\title{
Article
}

\section{DOA Estimation in Low SNR Environment through Coprime Antenna Arrays: An Innovative Approach by Applying Flower Pollination Algorithm}

\author{
Khurram Hameed ${ }^{1, *}$, Shanshan Tu ${ }^{2, *}{ }^{-}$, Nauman Ahmed ${ }^{3}{ }^{(0)}$, Wasim Khan ${ }^{1}$, Ammar Armghan ${ }^{4, *}$,

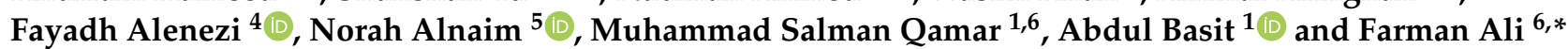 \\ check for \\ 1 Department of Electrical Engineering, International Islamic University, Islamabad 44000, Pakistan; \\ wasim.khan@iiu.edu.pk (W.K.); mskhan131@qurtuba.edu.pk (M.S.Q.); abdulbasit@iiu.edu.pk (A.B.) \\ 2 Engineering Research Center of Intelligent Perception and Autonomous Control, Faculty of Information \\ Technology, Beijing University of Technology, Beijing 100124, China \\ 3 School of Marine Science and Technology, Northwestern Polytechnical University, Xi'an 710072, China; \\ nauman@mail.nwpu.edu.cn \\ 4 Department of Electrical Engineering, College of Engineering, Jouf University, Sakaka 72388, Saudi Arabia; \\ fshenezi@ju.edu.sa \\ 5 Department of Computer Science, College of Sciences and Humanities in Jubail, Imam Abdulrahman Bin \\ Faisal University, Dammam 31441, Saudi Arabia; Nmalnaim@iau.edu.sa \\ 6 Department of Electrical Engineering, Qurtuba University of Science and IT, Dera Ismail Khan 29050, Pakistan \\ * Correspondence: khurram.phdee112@iiu.edu.pk (K.H.); sstu@bjut.edu.cn (S.T.); aarmghan@ju.edu.sa (A.A.); \\ drfarmanali.optics@qurtuba.edu.pk (F.A.)
}

updates

Citation: Hameed, K.; Tu, S.; Khan, W.; Ahmed, N.; Armghan, A.;

Alenezi, F.; Alnaim, N.; Qamar, M.S.; Basit, A.; Ali, F. DOA Estimation in Low SNR Environment through Coprime Antenna Arrays: An Innovative Approach by Applying Flower Pollination Algorithm. Appl. Sci. 2021, 11, 7985. https://doi.org/ 10.3390/app11177985

Academic Editor: Andrea Prati

Received: 27 July 2021

Accepted: 25 August 2021

Published: 29 August 2021

Publisher's Note: MDPI stays neutral with regard to jurisdictional claims in published maps and institutional affiliations.

Copyright: (C) 2021 by the authors Licensee MDPI, Basel, Switzerland. This article is an open access article distributed under the terms and conditions of the Creative Commons Attribution (CC BY) license (https:// creativecommons.org/licenses/by/ $4.0 /)$.
Abstract: The design of the modern computing paradigm of heuristics is an innovative development for parameter estimation of direction of arrival (DOA) using sparse antenna arrays. In this study, the optimization strength of the flower pollination algorithm (FPA) is exploited for the DOA estimation in a low signal to noise ratio (SNR) regime by applying coprime sensor arrays (CSA). The enhanced degree of freedom (DOF) is achieved with FPA by investigating the global minima of highly nonlinear cost function with multiple local minimas. The sparse structure of CSA demonstrates that the DOF up to $O(M N)$ is achieved by employing $M+N$ CSA elements, where $M$ and $N$ are the numbers of antenna elements used to construct the CSA. Performance analysis is conducted for estimation accuracy, robustness against noise, robustness against snapshots, frequency distribution of root mean square error (RMSE), variability analysis of RMSE, cumulative distribution function (CDF) of RMSE over Monte Carlo runs and the comparative studies of particle swarm optimization (PSO). These reveal the worth of the proposed methodology for estimating DOA.

Keywords: direction of arrival (DOA); coprime sensor array (CSA); flower pollination algorithm (FPA); particle swarm optimization (PSO); additive white gaussian noise (AWGN); virtual uniform linear array (vULA)

\section{Introduction}

Accurate estimation of target locations in array signal processing has been received a great acclaim in military and commercial applications like radar [1,2] sonar [3], seismology [4] and mobile communications [5,6]. Direction of arrival estimation and spatial filtering are becoming two important hotspots in the field of array signal processing. In accordance with literature, DOA estimation can be categorized into two important aspects with respect to estimation:the first is distribution array structures and the second one is estimation algorithms. The array structure is divided into two major groups depending upon the distance between the antenna elements. First are the uniform linear arrays ULA [7] and second is the sparse arrays structure [8-10]. A vast amount of literature is available on ULAs. In ULAs, all antenna elements are equispaced and furthermore, distance between 
each antenna element is equal to a fraction of wavelength of the received signal. To detect more targets using ULA, more antenna elements will be needed, which will increase the cost of hardware and computing complexity. This limitation in ULAs leads us to the world of sparse arrays.

Minimum redundancy array (MRA) [11], nested array (NA) [12,13] and coprime sensor arrays (CSA) [14,15] are the most popular types of the sparse arrays. These spare arrays are used to attain more number of degree of freedom rather than $\mathrm{N}-1$ targets with $\mathrm{N}$ sensors. In sparse arrays, distance between each antenna elements is not same. MRA has maximum $N(N-1)+1$ DOF but it has no closed form expression for its array structure. A nested array has $\mathrm{O}\left(\mathrm{N}^{2}\right)$ DOF but it has more mutual coupling issue than MRA and coprime due to a dense ULA in its physical structure. Although, CSA has more holes in its co-array structure but it has less mutual coupling and additionally, $O(M N)$ is the DOF for this array structure. The past decade has been shown a huge growth in CSAs. Consequently, CSA is used in this paper due to enhanced DOF and reduced mutual coupling effect for efficient detection of targets in far field. CSA is obtained from union of two ULAs of coprime numbers.

The second aspect of the DOA estimation is categorized into two further parts. First are the deterministic algorithms and the second part is the heuristic algorithms. Deterministic algorithms are sub-space based and consist of multiple signal classification(MUSIC) [16], root MUSIC [17], estimation of signal parameters via rotational invariance techniques (ESPRIT) [18], minimum variance distortionless response (MVDR) [19], maximum Likelihood (ML) [20], etc. Although, these sub-space methods perform well and provide better resolution [21,22] in terms of computational complexity, solving a problem quickly and increasing robustness to also increase efficiency, the degree of freedom heuristic algorithms are employed. In consonance with the literature, sub-space based methods are eligible to estimate $N-1$ targets by configuring $\mathrm{N}$ antenna elements. Therefore, heuristic algorithms are used to subdue this issue. Numerous algorithms are included under heuristic algorithms, such as Genetic Algorithm (GA) [23], flower pollination algorithm (FPA) [24], particle swarm optimization (PSO) [25,26], ant colony optimization (ACO) [27], bee colony optimization (BCO) [28], simulated annealing (SA) [29], differential evolution (DE) [30], etc. For far-field narrow-band signal DOA estimation, so far, a set of algorithms have been proposed, such as Estimation of Signal Parameters via Rotational Invariance Techniques (ESPRIT), Multiple Signal Classification (MUSIC) , Maximum Likelihood (ML, including Deterministic ML (DML) and Stochastic ML (SML), compressed sensing, and Weighted Subspace Fitting (WSF). Among these algorithms, it is well known that ML and WSF have the highest accuracy of DOA estimation. However, it is also well demonstrated that the computational complexity of them is also the highest because their criteria are nonlinear multi-dimensional optimization [31] . In this research work, we exploit the features of Flower Pollination Algorithm and estimate the direction of arrival. FPA is a biologicalinspired soft computing algorithm, which is associated with the transformation of pollens by pollinators like bees, birds and other insects. There are two basic procedures for transformation of pollens. The first one is biotic and the other is an abiotic process. Biotic or cross-pollination is also known as global search pollination and abiotic or self- pollination is also known as local search pollination. In FPA the major objective is to deduce the best reproduction of plants by surviving the fittest flower in the flowering plants [32]. The aim of our research is to estimate DOA through novel computing paradigm using coprime sensor array with the help of meta heuristic technique. We analyze the performance of the proposed scheme in term of estimation accuracy, robustness against noise, robustness against snapshots, frequency distribution of RMSE, variability analysis of RMSE and CDF of RMSE over Monte Carlo runs.

\section{Organization and Notation of Paper}

The rest of the paper is organized as follows. A mathematical model of the proposed scheme is derived in Section 2, Section 3 elaborates on the frame work of the FPA and 
PSO, results and discussion are explained in Section 4 and finally, the conclusion of the presented model is concluded in Section 5 .

\section{Mathematical Modeling}

Suppose signals of $L$ narrowband uncorrelated targets are impinging on CSA consisting of two sub ULAs. Sub-array 1 contains $M$ antenna elements having $N d$ space among each antenna element as shown in Figure 1a while sub-array 2 has $N$ antenna elements $M d$ apart from each other as shown in Figure $1 b . M$ and $N$ are coprime integers and $d$ is equal to the half of the wave length $(\lambda / 2)$. By combining these two sub arrays, we get a generalized coprime antenna array having $M+N-1$ elements, as seen in Figure 1c. The first antenna element is shared with both sub-ULAs and physical location of each antenna element of CSA is enclosed in Equation (1) .

$$
I=\{0, N d, M d, 2 N d, 2 M d, \ldots,(N-1) M d,(M-1) N d\}
$$

Direction of arrival of $L$ uncorrelated signals is summarized in vector $\theta$

$$
\theta=\left[\theta_{1}, \theta_{2}, \theta_{3}, \ldots, \theta_{L}\right]
$$

Hence, the received signal of $\mathrm{L}$ uncorrelated targets impinging on coprime array is given by

$$
x(t)=\sum_{l=1}^{L} a_{l}(\theta) s_{l}(t)+n(t)=A(\theta) s(t)+n(t)
$$

where

$$
\begin{gathered}
A(\theta)=\left[a_{1}(\theta), a_{2}(\theta), \ldots, a_{L}(\theta)\right] \\
a_{l}(\theta)=\left[1, e^{\pi N \sin \theta_{l}}, e^{\pi M \sin \theta_{l}}, e^{\pi 2 N \sin \theta_{l}}, e^{\pi 2 M \sin \theta_{l}}, \ldots, e^{\pi(N-1) M d \sin \theta_{l}}, e^{\pi(M-1) N d \sin \theta_{l}}\right] \\
s_{l}(t)=\left[s_{1}(t), s_{2}(t), \ldots, s_{L}(t)\right]^{T}
\end{gathered}
$$

and $n(t)$ is the iid and complex additive white Gaussian noise (AWGN) with variance $\sigma I^{2}$ and zero mean.

$$
n(t)=\left[n_{1}(t), n_{2}(t), \ldots n_{M+N-1}(t)\right]
$$

Based on this coprime array structure, we can be performed the computation of covariance matrix by taking expectation of the received signal as presented in following equation

$$
R x x=E\left[x(t) x^{H}(t)\right]
$$

By solving this equation we will get

$$
R x x=A R s s A^{H}+\sigma^{2} I
$$

Rss is the amplitude covariance matrix of received signal as shown in following Eq (9)

$$
R s s=E\left[s(t) s^{H}(t)\right]
$$

Now virtual uniform linear array can be obtained by performing vectorization on covariance matrix $(R x x)$ as expressed in the following equation

$$
z=\operatorname{vec}(R x x)=\operatorname{vec}\left(A R s s A^{H}+\sigma^{2} I\right)=D \sigma^{2}+\sigma^{2} I
$$

where $D$ is the Kronecker product and the elements in $D$ are expressed in the form of following equation

$$
e^{j K\left(I_{i}-I_{j}\right) \sin \theta_{l} \text { where } i, j=0,1,2, \ldots, M+N-1} .
$$


In the process of vectorization, all columns of correlation matrix are placed one by one under the first column. After performing vectorization, the correlation matrix become a column vector $z^{(M+N-1)^{2} \times 1}$. This column vector $z$ is sorted out in accordance with exponent term of the received signal. After sorting, redundant terms of this sorted vector are being skipped. In [33], this can be assessed as continuous virtual uniform linear array (vULA)

$$
I_{c}=\{(M n d-N m d) \cup(N m d-M n d)\}, \text { where } 0<m<M-1 \text { and } 0<n<N-1
$$

Locations of this vULA elements can be expressed as

$$
I_{v}=\left\{\left(I_{i}-I_{j}\right) i, j=0,1,2, \ldots, M+N-1\right\}
$$

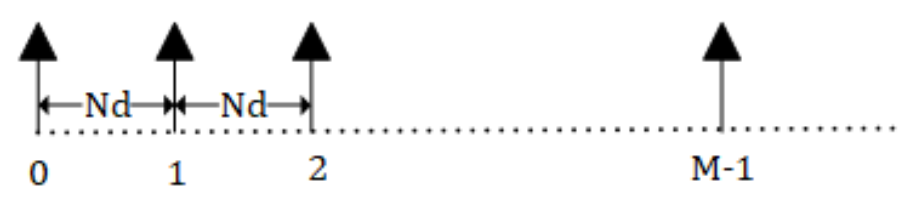

(a)

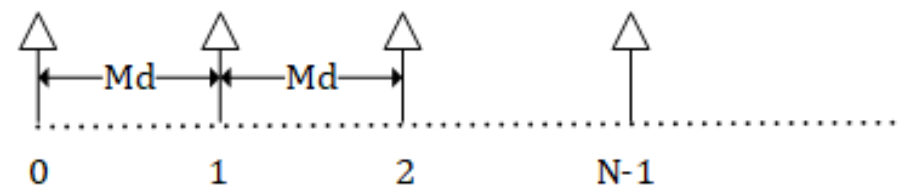

(b)

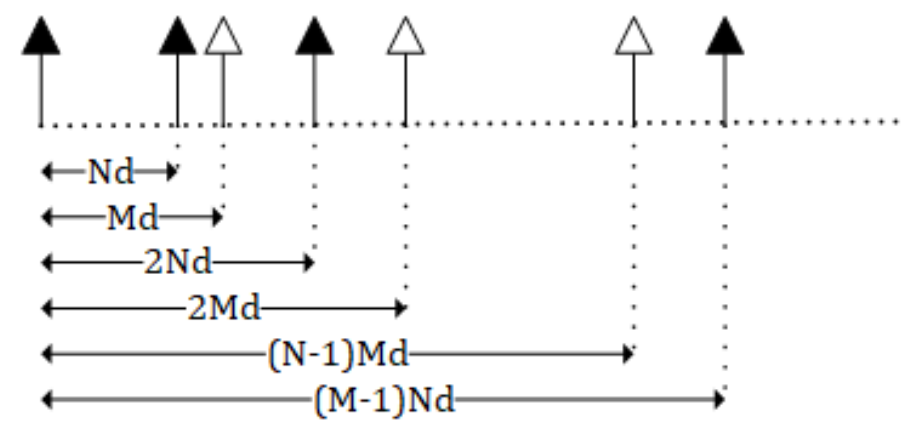

(c)

Figure 1. Coprime antenna array configuration: (a) sub antenna array 1; (b) sub antenna array 2; (c) generalize coprime antenna array.

We concentrate on the continuous part of vULA of CSA for application of our proposed algorithms. Due to this continuous part of the vULA, we get a higher degree of freedom and this DOF is directly proportional to the length of continuous vULA.

\section{Proposed Methodology}

\subsection{Flower Pollination Algorithm}

In this research article, a flower pollination algorithm is designed to optimize the received signal at coprime antenna arrays. In Figure 2, a flow chart of FPA is illustrated to describe the complete function of FPA. Briefly,the flower pollination algorithm is described in following steps. 


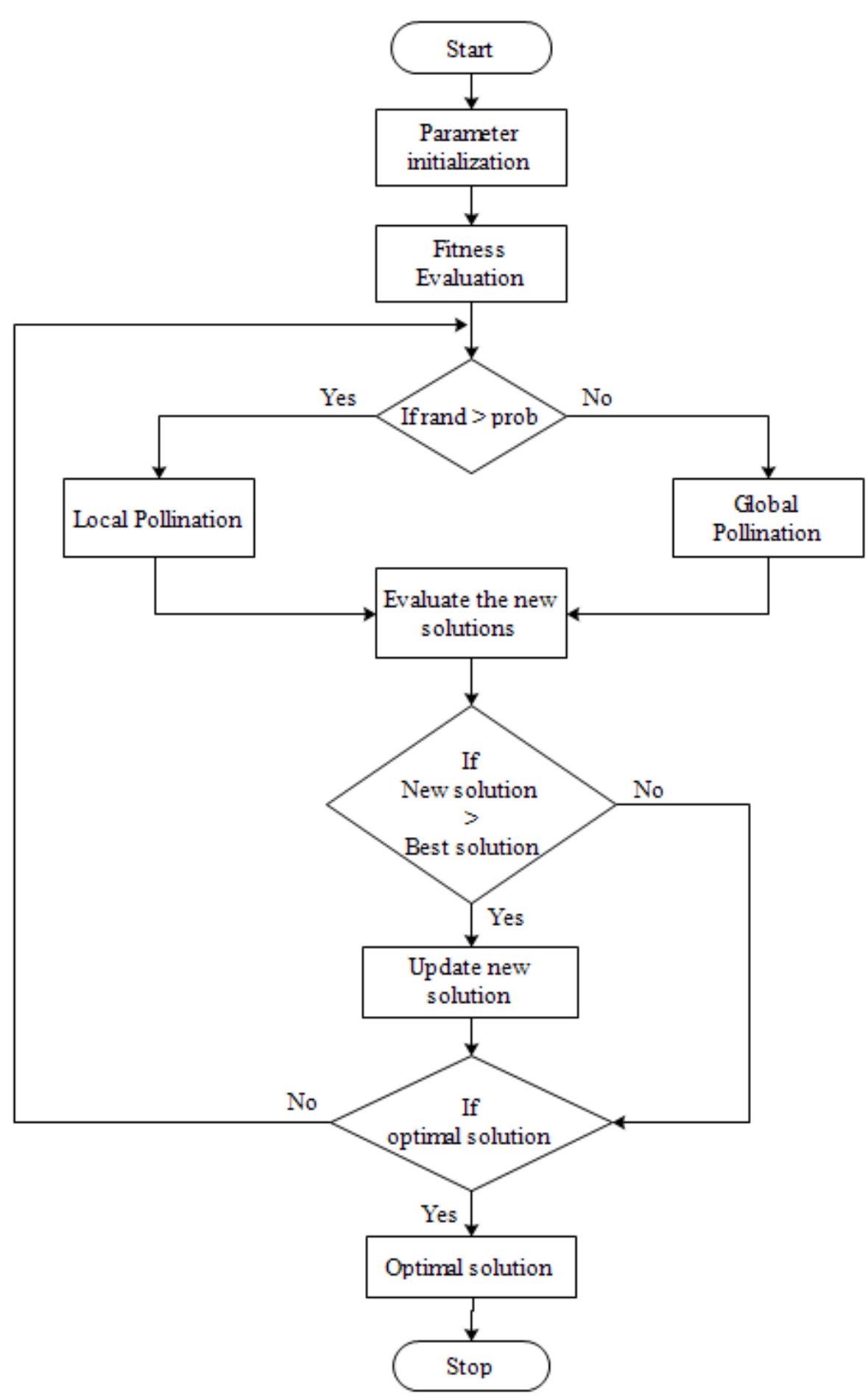

Figure 2. Flow diagram for Flower Pollination Algorithm.

Step 1: Population Generation

Randomly generate the initial population $x_{i}$.

Then evaluate the whole population by the help of fitness function $f\left(x_{i}\right)$.

Step 2: Global pollination process

By using Levy distribution, global pollination process is done by executing the following equation

$$
x_{i}^{(t+1)}=x_{i}^{t}+L\left(g^{*}-x_{i}^{t}\right)
$$

Step 3: Local pollination process 
Choose two solutions $\mathrm{i}$ and $\mathrm{j}$ randomly and generate the parameter $\epsilon$ for exploration process by executing following equation

$$
x_{i}^{(t+1)}=x_{i}^{t}+\epsilon\left(x_{j}^{t}-x_{k}^{t}\right)
$$

Step 4: Update solution

At the end we check that if new solution is better than the older one, then set

$$
x_{i}^{t+1}=x_{i}^{t+1}
$$

otherwise set

$$
x_{i}^{t+1}=x_{i}^{t}
$$

This process is repeated until the best solution is achieved.

\subsection{Particle Swarm Optimization}

Particle swarm optimization is a nature inspired robust stochastic optimization technique. The workings of PSO can be explained in following six steps as illustrated in Figure 3 Step 1: Initialization: solution.

The PSO algorithm is initialized by a group of random particles and each particle is a

Ater Initializing parameters $\left(x_{i}\right)$ and velocities $\left(v_{i}\right)$. Each particle searches for the optimal value by updating the generation.

In each iteration every particle is updated. The first best one is the best solution. After finding the best value particle, update its velocity and position again.

Particle updates its position by

$$
x_{i}^{(t+1)}=x_{i}^{(t)}+v_{i}^{(t)} * t
$$

Particle updates its velocity through following equation

$$
x_{i}^{(t+1)}=w * v_{i}^{(t)}+c_{1} * v_{1}\left(\text { xbesti } i^{t}-x_{i}^{t}\right)+c_{2} * r_{2}\left(\text { gbestit } x_{i}^{t}\right)
$$

where

$c_{1}$ is the constant parameter;

$r_{1}$ is the random parameter;

Xbest is the best particle position;

Gbest is the group best position.

Step 2: Evaluate Fitness:

Calculate fitness value for each particle. If fitness value is greater than the gbest then set new value as gbest and choose particle with the best fitness value.

Step 3: For each particle calculate velocity and position from Equations (1) and (2).

Step 4: Evaluate Fitness $f\left(x_{i}^{t}\right)$ and find current best.

Step 5: Update $t=t+1$.

Step 6: Output gbest and $x_{i}(t)$.

The process will be continued until the condition is met.

\subsection{Fitness Function}

Fitness function plays an important role in optimization problems. This is a fundamental tool for the evaluation of the population. This evaluation is performed in each iteration of the algorithm. Fitness function is the difference between the actual and estimated value and it is defined in the following equation

$$
f\left(x_{i}\right)=\left|x_{a_{i}}(t)-x_{e_{i}}(t)\right|^{2}
$$


In both methodologies, this fitness function is used for evaluation of pollens and particles.

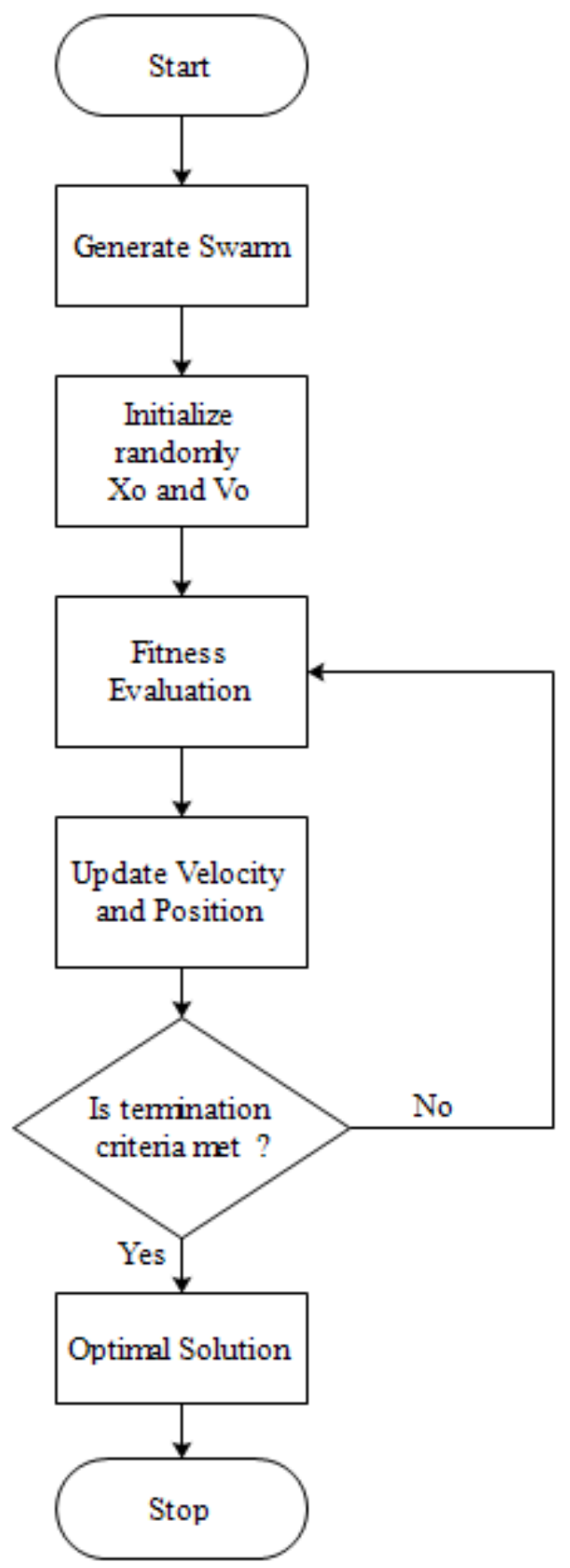

Figure 3. Flow diagram for Particle Swarm Optimization.

\section{Results, Discussions and Achievements}

This section contains various simulated results to validate the potential of FPA and PSO. This analysis is done in term of estimation accuracy, robustness against noise, RMSE vs. snapshots, variation analysis of RMSE, frequency distribution of RMSE, cumulative distribution function of RMSE and box plot analysis under different varying parameters of far field sources. In all of these simulations, Additive White Gaussian Noise (AWGN) channel is assumed with zero mean and variance $\sigma^{2}$ while physical coprime antenna elements are 6 and 200 snapshots are taken for each simulation. All simulated results are compared by estimating seven, nine and eleven targets which are lo- 
cated at $\left[20^{\circ}, 40^{\circ}, 60^{\circ}, 80^{\circ}, 100^{\circ}, 120^{\circ}, 140^{\circ}\right],\left[20^{\circ}, 40^{\circ}, 60^{\circ}, 80^{\circ}, 100^{\circ}, 120^{\circ}, 140^{\circ}, 160^{\circ} .170^{\circ}\right]$ and $\left[20^{0}, 35^{0}, 50^{\circ}, 65^{0}, 80^{\circ}, 95^{0}, 110^{0}, 120^{0}, 135^{0}, 150^{0}, 165^{0}\right]$ simultaneously.

\subsection{Estimation Accuracy}

Estimation accuracy of FPA and PSO is looked into by varying the number of sources and their positions. This analysis is set out by best, worst and mean values of the angles estimated by proposed methods to validate their performance along with statistical analysis. In the case of seven targets, we clearly monitored that angles estimated by FPA are accurate than PSO at $0 \mathrm{~dB},-5 \mathrm{~dB}$ and $-10 \mathrm{~dB}$ SNRs as depicted in Tables 1-3. Furthermore, Tables 1-3 includes the standard deviation (STD) analysis for the FPA and PSO models, which clearly define that the proposed model has optimized outcomes.

Table 1. Estimation accuracy and STD for seven targets at SNR $=0 \mathrm{~dB}$.

\begin{tabular}{ccccccccc}
\hline Actual Angles & & $\boldsymbol{\theta}_{\mathbf{1}}=\mathbf{2 0}$ & $\boldsymbol{\theta}_{\mathbf{2}}=\mathbf{4 0}$ & $\boldsymbol{\theta}_{\mathbf{3}}=\mathbf{6 0}$ & $\boldsymbol{\theta}_{\mathbf{4}}=\mathbf{8 0}$ & $\boldsymbol{\theta}_{\mathbf{5}}=\mathbf{1 0 0}$ & $\boldsymbol{\theta}_{\mathbf{6}}=\mathbf{1 2 0}$ & $\boldsymbol{\theta}_{\mathbf{7}}=\mathbf{1 4 0}$ \\
\hline \multirow{4}{*}{ FPA } & Best & 20.554 & 39.328 & 59.914 & 79.794 & 100.400 & 120.194 & 139.941 \\
& Mean & 20.184 & 36.820 & 58.325 & 78.922 & 102.355 & 121.292 & 142.981 \\
& Worst & 180.000 & 37.021 & 59.478 & 80.592 & 100.762 & 119.896 & 139.453 \\
& STD & 8.0112 & 7.6306 & 7.3363 & 7.2551 & 7.076 & 6.7608 & 13.0759 \\
\hline \multirow{3}{*}{ PSO } & Best & 22.585 & 37.755 & 59.198 & 79.539 & 96.225 & 106.215 & 120.000 \\
& Mean & 23.146 & 34.461 & 52.488 & 63.898 & 80.206 & 100.494 & 119.387 \\
& Worst & 0.000 & 36.178 & 37.319 & 58.481 & 58.711 & 78.657 & 98.202 \\
& STD & 8.8477 & 5.3369 & 10.351 & 15.299 & 19.8711 & 21.992 & 23.3032 \\
\hline
\end{tabular}

Table 2. Estimation accuracy and STD for seven targets at $\mathrm{SNR}=-5 \mathrm{~dB}$.

\begin{tabular}{ccccccccc}
\hline Actual Angles & & $\boldsymbol{\theta}_{\mathbf{1}}=\mathbf{2 0}$ & $\boldsymbol{\theta}_{\mathbf{2}}=\mathbf{4 0}$ & $\boldsymbol{\theta}_{\mathbf{3}}=\mathbf{6 0}$ & $\boldsymbol{\theta}_{\mathbf{4}}=\mathbf{8 0}$ & $\boldsymbol{\theta}_{\mathbf{5}}=\mathbf{1 0 0}$ & $\boldsymbol{\theta}_{\mathbf{6}}=\mathbf{1 2 0}$ & $\boldsymbol{\theta}_{\mathbf{7}}=\mathbf{1 4 0}$ \\
\hline \multirow{4}{*}{ FPA } & Best & 20.554 & 39.328 & 59.914 & 79.794 & 100.400 & 120.194 & 139.941 \\
& Mean & 14.697 & 38.478 & 59.783 & 80.211 & 98.590 & 121.139 & 141.271 \\
& Worst & 180.000 & 34.132 & 60.093 & 78.950 & 101.367 & 119.340 & 139.785 \\
& STD & 8.5051 & 8.1359 & 7.6892 & 7.8932 & 7.6953 & 7.3345 & 13.8623 \\
\hline \multirow{3}{*}{ PSO } & Best & 24.320 & 39.690 & 59.613 & 79.500 & 98.327 & 110.181 & 120.000 \\
& Mean & 24.777 & 32.753 & 51.729 & 63.982 & 80.608 & 100.351 & 119.243 \\
& Worst & 0.261 & 35.533 & 36.028 & 55.937 & 60.200 & 78.614 & 98.036 \\
& STD & 9.7167 & 5.8089 & 11.1897 & 15.428 & 19.3838 & 20.5064 & 21.6453 \\
\hline
\end{tabular}

Table 3. Estimation accuracy and STD for seven targets at SNR $=-10 \mathrm{~dB}$.

\begin{tabular}{ccccccccc}
\hline Actual Angles & & $\boldsymbol{\theta}_{\mathbf{1}}=\mathbf{2 0}$ & $\boldsymbol{\theta}_{\mathbf{2}}=\mathbf{4 0}$ & $\boldsymbol{\theta}_{\mathbf{3}}=\mathbf{6 0}$ & $\boldsymbol{\theta}_{\mathbf{4}}=\mathbf{8 0}$ & $\boldsymbol{\theta}_{\mathbf{5}}=\mathbf{1 0 0}$ & $\boldsymbol{\theta}_{\mathbf{6}}=\mathbf{1 2 0}$ & $\boldsymbol{\theta}_{\mathbf{7}}=\mathbf{1 4 0}$ \\
\hline \multirow{4}{*}{ FPA } & Best & 21.034 & 39.874 & 59.772 & 80.466 & 99.778 & 120.534 & 139.494 \\
& Mean & 25.846 & 34.286 & 58.860 & 76.878 & 102.507 & 120.626 & 144.842 \\
& Worst & 179.201 & 31.046 & 58.433 & 76.493 & 103.240 & 121.604 & 145.273 \\
& STD & 7.3575 & 7.0672 & 5.51 & 6.1898 & 5.5168 & 5.5472 & 10.4078 \\
\hline \multirow{3}{*}{ PSO } & Best & 16.144 & 34.875 & 57.315 & 78.143 & 102.429 & 120.000 & 120.000 \\
& Mean & 29.010 & 36.222 & 52.800 & 62.549 & 77.717 & 101.883 & 120.000 \\
& Worst & 0.000 & 32.768 & 33.258 & 40.144 & 58.502 & 78.027 & 99.566 \\
& STD & 12.9084 & 6.3004 & 15.559 & 17.1445 & 20.1051 & 19.7943 & 21.6932 \\
\hline
\end{tabular}

The statistical analysis including STD for nine targets are declared in Tables 4-6, comparing the performance of FPA and PSO algorithms. The outcomes prove that the computation analysis of FPA is more optimized than PSO. 
Table 4. Estimation accuracy and STD for nine targets at SNR $=0 \mathrm{~dB}$.

\begin{tabular}{ccccccccccc}
\hline Actual Angles & & $\boldsymbol{\theta}_{\mathbf{1}}=\mathbf{2 0}$ & $\boldsymbol{\theta}_{\mathbf{2}}=\mathbf{4 0}$ & $\boldsymbol{\theta}_{\mathbf{3}}=\mathbf{6 0}$ & $\boldsymbol{\theta}_{\mathbf{4}}=\mathbf{8 0}$ & $\boldsymbol{\theta}_{\mathbf{5}}=\mathbf{1 0 0}$ & $\boldsymbol{\theta}_{\mathbf{6}}=\mathbf{1 2 0}$ & $\boldsymbol{\theta}_{\mathbf{7}}=\mathbf{1 4 0}$ & $\boldsymbol{\theta}_{\mathbf{8}}=\mathbf{1 6 0}$ & $\boldsymbol{\theta}_{\mathbf{9}}=\mathbf{1 7 0}$ \\
\hline \multirow{4}{*}{ FPA } & Best & 21.006 & 38.750 & 60.502 & 79.982 & 100.441 & 119.939 & 140.742 & 160.451 & 170.148 \\
& Mean & 0.215 & 39.874 & 60.583 & 79.703 & 100.416 & 119.783 & 142.500 & 179.858 & 179.915 \\
& Worst & 0.000 & 38.758 & 58.943 & 80.572 & 100.388 & 120.260 & 142.292 & 1.398 & 0.000 \\
& STD & 14.2678 & 18.1622 & 14.7291 & 14.3368 & 14.2258 & 13.776 & 13.9762 & 13.5198 & 9.3947 \\
\hline \multirow{3}{*}{ PSO } & Best & 1.186 & 37.058 & 59.335 & 82.653 & 96.478 & 120.000 & 104.940 & 75.794 & 0.000 \\
& Mean & 31.074 & 41.587 & 55.661 & 80.701 & 100.348 & 118.800 & 65.155 & 0.000 & 0.000 \\
& Worst & 38.524 & 38.120 & 56.432 & 79.084 & 98.278 & 0.339 & 0.000 & 62.001 & 0.000 \\
& STD & 19.9584 & 39.6766 & 41.721 & 38.3223 & 44.4625 & 50.1277 & 56.1085 & 59.8789 & 52.0099 \\
\hline
\end{tabular}

Table 5. Estimation accuracy and STD for nine targets at SNR $=-5 \mathrm{~dB}$.

\begin{tabular}{ccccccccccc}
\hline Actual Angles & & $\boldsymbol{\theta}_{\mathbf{1}}=\mathbf{2 0}$ & $\boldsymbol{\theta}_{\mathbf{2}}=\mathbf{4 0}$ & $\boldsymbol{\theta}_{\mathbf{3}}=\mathbf{6 0}$ & $\boldsymbol{\theta}_{\mathbf{4}}=\mathbf{8 0}$ & $\boldsymbol{\theta}_{\mathbf{5}}=\mathbf{1 0 0}$ & $\boldsymbol{\theta}_{\mathbf{6}}=\mathbf{1 2 0}$ & $\boldsymbol{\theta}_{\mathbf{7}}=\mathbf{1 4 0}$ & $\boldsymbol{\theta}_{\mathbf{8}}=\mathbf{1 6 0}$ & $\boldsymbol{\theta}_{\mathbf{9}}=\mathbf{1 7 0}$ \\
\hline \multirow{4}{*}{ FPA } & Best & 20.063 & 40.167 & 59.702 & 79.861 & 100.205 & 120.889 & 140.965 & 162.774 & 171.189 \\
& Mean & 23.035 & 34.792 & 57.209 & 80.132 & 100.174 & 116.792 & 129.355 & 154.086 & 156.307 \\
& Worst & 11.371 & 36.931 & 59.788 & 78.922 & 99.862 & 120.054 & 134.764 & 151.199 & 0.250 \\
& STD & 10.8943 & 14.1462 & 13.4699 & 13.8556 & 14.1729 & 13.4184 & 13.5677 & 13.3974 & 9.9573 \\
\hline \multirow{3}{*}{ PSO } & Best & 0.885 & 36.881 & 59.177 & 79.224 & 97.356 & 120.000 & 120.000 & 105.922 & 0.000 \\
& Mean & 31.074 & 41.587 & 55.661 & 80.701 & 100.348 & 118.800 & 65.155 & 0.000 & 0.000 \\
& Worst & 38.524 & 38.120 & 56.432 & 79.084 & 98.278 & 0.339 & 0.000 & 62.001 & 0.000 \\
& STD & 19.9206 & 39.5109 & 31.5467 & 36.5097 & 42.1808 & 48.2541 & 54.0918 & 57.903 & 50.7356 \\
\hline
\end{tabular}

Table 6. Estimation accuracy and STD for nine targets at SNR $=-10 \mathrm{~dB}$.

\begin{tabular}{ccccccccccc}
\hline Actual Angles & & $\boldsymbol{\theta}_{\mathbf{1}}=\mathbf{2 0}$ & $\boldsymbol{\theta}_{\mathbf{2}}=\mathbf{4 0}$ & $\boldsymbol{\theta}_{\mathbf{3}}=\mathbf{6 0}$ & $\boldsymbol{\theta}_{\mathbf{4}}=\mathbf{8 0}$ & $\boldsymbol{\theta}_{\mathbf{5}}=\mathbf{1 0 0}$ & $\boldsymbol{\theta}_{\mathbf{6}}=\mathbf{1 2 0}$ & $\boldsymbol{\theta}_{\mathbf{7}}=\mathbf{1 4 0}$ & $\boldsymbol{\theta}_{\mathbf{8}}=\mathbf{1 6 0}$ & $\boldsymbol{\theta}_{\mathbf{9}}=\mathbf{1 7 0}$ \\
\hline \multirow{3}{*}{ FPA } & Best & 18.168 & 38.385 & 59.174 & 79.491 & 101.673 & 119.234 & 140.863 & 158.025 & 170.230 \\
& Mean & 0.000 & 32.733 & 58.255 & 77.206 & 101.260 & 117.178 & 127.036 & 148.510 & 169.016 \\
& Worst & 11.230 & 38.897 & 61.967 & 79.402 & 100.536 & 119.506 & 143.753 & 2.138 & 0.000 \\
& STD & 11.5865 & 15.2439 & 15.913 & 15.1088 & 15.3368 & 13.0878 & 13.8784 & 12.2759 & 11.0899 \\
\hline \multirow{3}{*}{ PSO } & Best & 0.371 & 36.758 & 59.827 & 79.031 & 97.880 & 120.000 & 120.000 & 106.209 & 0.000 \\
& Mean & 29.041 & 41.913 & 59.563 & 80.782 & 102.079 & 119.840 & 73.947 & 0.000 & 0.000 \\
& Worst & 37.783 & 37.524 & 59.243 & 78.662 & 99.615 & 58.886 & 0.000 & 0.000 & 0.000 \\
& STD & 19.9239 & 39.508 & 40.2039 & 36.9535 & 42.1866 & 48.3715 & 55.2631 & 58.0799 & 51.2382 \\
\hline
\end{tabular}

Tables 7-9 illustrate the analysis of different angles in terms of best, mean, worst and STD for FPA and PSO algorithms. By keeping in view the analysis of STD, it clearly observed that the achievements of FPA are much better than PSO.

Table 7. Estimation accuracy and STD for eleven targets at SNR $=0 \mathrm{~dB}$.

\begin{tabular}{|c|c|c|c|c|c|c|c|c|c|c|c|c|}
\hline Actual Angles & & $\theta_{1}=20$ & $\theta_{2}=35$ & $\theta_{3}=50$ & $\theta_{4}=65$ & $\theta_{5}=80$ & $\theta_{6}=95$ & $\theta_{7}=110$ & $\theta_{8}=120$ & $\theta_{9}=135$ & $\theta_{10}=150$ & $\theta_{11}=165$ \\
\hline \multirow{4}{*}{ FPA } & Best & 20.399 & 34.092 & 50.469 & 66.942 & 80.878 & 95.569 & 109.970 & 118.790 & 134.319 & 150.301 & 160.555 \\
\hline & Mean & 17.832 & 20.145 & 40.542 & 55.367 & 73.083 & 89.377 & 104.883 & 115.665 & 124.661 & 145.335 & 149.661 \\
\hline & Worst & 2.486 & 28.774 & 44.332 & 58.798 & 74.446 & 89.797 & 103.624 & 118.702 & 136.652 & 148.535 & 0.257 \\
\hline & STD & 12.8879 & 13.0721 & 9.6502 & 10.2882 & 10.2654 & 10.3802 & 10.0996 & 8.9518 & 9.696 & 9.9422 & 12.3896 \\
\hline \multirow{4}{*}{ PSO } & Best & 33.810 & 45.343 & 60.019 & 73.433 & 82.736 & 92.641 & 112.055 & 120.000 & 101.111 & 0.000 & 0.000 \\
\hline & Mean & 1.489 & 32.933 & 54.975 & 65.939 & 75.908 & 97.667 & 110.797 & 119.215 & 85.444 & 42.657 & 0.000 \\
\hline & Worst & 0.653 & 38.887 & 39.501 & 63.090 & 83.131 & 74.659 & 96.677 & 114.281 & 55.525 & 0.489 & 0.276 \\
\hline & STD & 19.8683 & 34.3679 & 23.1016 & 23.4132 & 27.2577 & 30.5871 & 34.19 & 33.4794 & 37.3056 & 39.7499 & 45.8505 \\
\hline
\end{tabular}


Table 8. Estimation accuracy and STD for eleven targets at SNR $=-5 \mathrm{~dB}$.

\begin{tabular}{|c|c|c|c|c|c|c|c|c|c|c|c|c|}
\hline Actual Angles & & $\theta_{1}=20$ & $\theta_{2}=35$ & $\theta_{3}=50$ & $\theta_{4}=65$ & $\theta_{5}=80$ & $\theta_{6}=95$ & $\theta_{7}=110$ & $\theta_{8}=120$ & $\theta_{9}=135$ & $\theta_{10}=150$ & $\theta_{11}=165$ \\
\hline \multirow{4}{*}{ FPA } & Best & 23.21 & 34.09 & 51.73 & 66.56 & 80.511 & 94.689 & 109.868 & 121.81 & 136.79 & 150.969 & 165.004 \\
\hline & Mean & 2.178 & 31.737 & 48.094 & 67.517 & 83.290 & 98.378 & 112.733 & 121.49 & 137.75 & 143.524 & 180 \\
\hline & Worst & 2.998 & 34.678 & 40.895 & 60.643 & 76.488 & 93.857 & 107.342 & 115.7 & 127.69 & 143.557 & 0.603 \\
\hline & STD & 11.2746 & 10.9455 & 9.9859 & 9.763 & 9.5543 & 9.17 & 7.709 & 6.8242 & 8.9132 & 10.3684 & 11.8853 \\
\hline \multirow{4}{*}{ PSO } & Best & 0.000 & 32.31 & 41.51 & 68.749 & 80.010 & 94.0313 & 106.08 & 120 & 114.28 & 57.657 & 0.977 \\
\hline & Mean & 0.601 & 32.904 & 55.206 & 66.032 & 77.191 & 90.0479 & 112.28 & 119.11 & 101.44 & 40.682 & 0.703 \\
\hline & Worst & 38.766 & 38.310 & 57.192 & 64.507 & 82.03 & 96.142 & 114.12 & 73.873 & 2.232 & 1.491 & 0.000 \\
\hline & STD & 19.7599 & 34.0793 & 18.3588 & 25.726 & 25.3566 & 29.5459 & 33.0406 & 29.977 & 33.9386 & 37.9637 & 45.2615 \\
\hline
\end{tabular}

Table 9. Estimation accuracy and STD for eleven targets at SNR $=-10 \mathrm{~dB}$.

\begin{tabular}{|c|c|c|c|c|c|c|c|c|c|c|c|c|}
\hline Actual Angles & & $\theta_{1}=20$ & $\theta_{2}=35$ & $\theta_{3}=50$ & $\theta_{4}=65$ & $\theta_{5}=80$ & $\theta_{6}=95$ & $\theta_{7}=110$ & $\theta_{8}=120$ & $\theta_{9}=135$ & $\theta_{10}=150$ & $\theta_{11}=165$ \\
\hline \multirow{4}{*}{ FPA } & Best & 19.25 & 36.78 & 51.86 & 64.31 & 79.67 & 96.18 & 110.49 & 120.45 & 135.79 & 153.02 & 166.20 \\
\hline & Mean & 28.37 & 34.49 & 55.83 & 72.93 & 88.82 & 104.98 & 116.67 & 125.51 & 144.95 & 153.74 & 179.04 \\
\hline & Worst & 29.76 & 37.34 & 55.15 & 72.85 & 89.77 & 106.00 & 0.25 & 121.24 & 143.21 & 146.09 & 0.000 \\
\hline & STD & 12.1514 & 11.0382 & 10.2101 & 10.6042 & 11.3763 & 11.875 & 7.0773 & 7.7574 & 9.3693 & 10.463 & 11.8565 \\
\hline \multirow{4}{*}{ PSO } & Best & 0.00 & 30.76 & 40.60 & 65.41 & 83.42 & 95.62 & 106.44 & 120 & 114.23 & 74.80 & 55.77 \\
\hline & Mean & 0.00 & 34.07 & 39.52 & 64.19 & 84.78 & 99.33 & 111.87 & 117.29 & 73.77 & 53.86 & 0.00 \\
\hline & Worst & 38.82 & 38.12 & 38.20 & 60.94 & 75.77 & 58.90 & 111.06 & 117.28 & 0.13 & 0.26 & 0.41 \\
\hline & STD & 19.7866 & 33.973 & 21.9043 & 25.7832 & 28.1443 & 31.7251 & 36.0186 & 33.9977 & 34.4507 & 38.2707 & 46.2669 \\
\hline
\end{tabular}

\subsection{Robustness against Noise}

Robustness against noise is one of the best analysis to measure the performance of algorithms used for DOA estimation. In this analysis, as signal to noise ratio increases, the value of RMSE decreases. RMSE is the performance indicator against different numbers of SNR in this analysis. In all three cases, FPA shows much robustness against noise than PSO as shown in Figures 4-6.

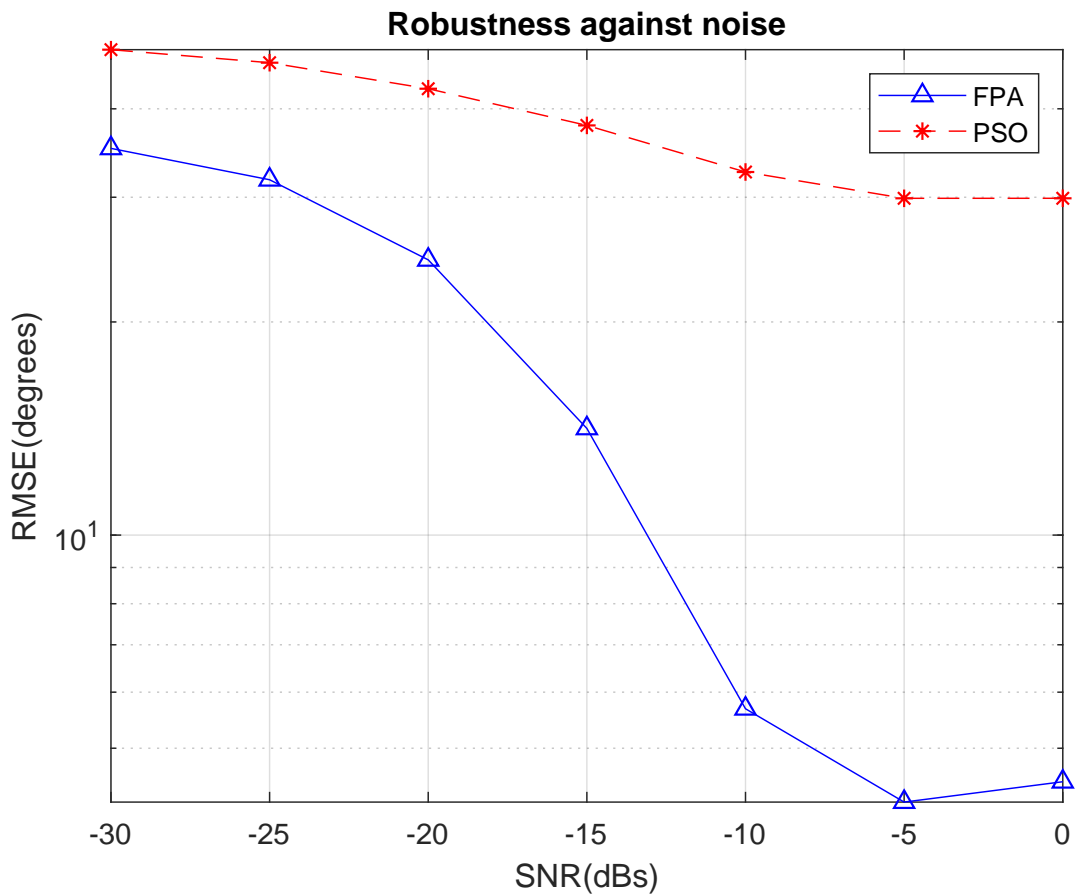

Figure 4. Robustness against noise for seven targets. 


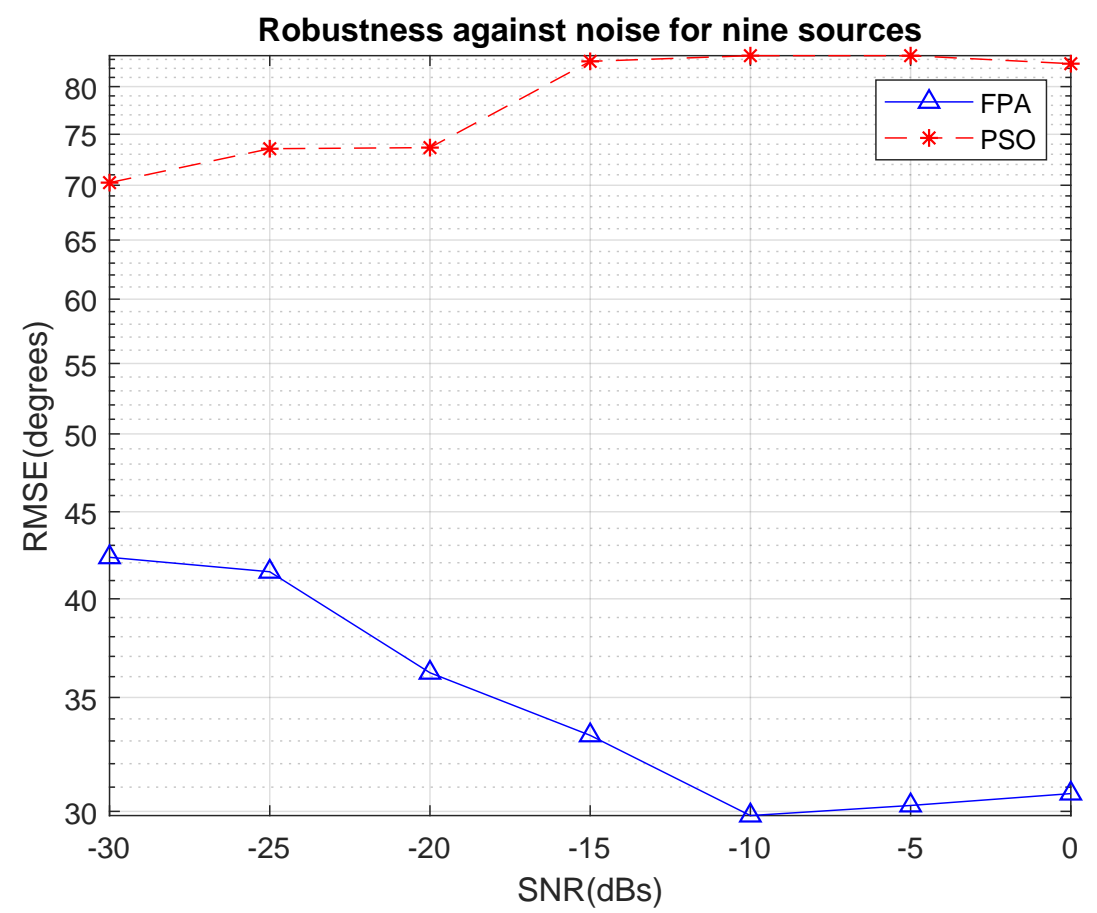

Figure 5. Robustness against noise for nine targets.

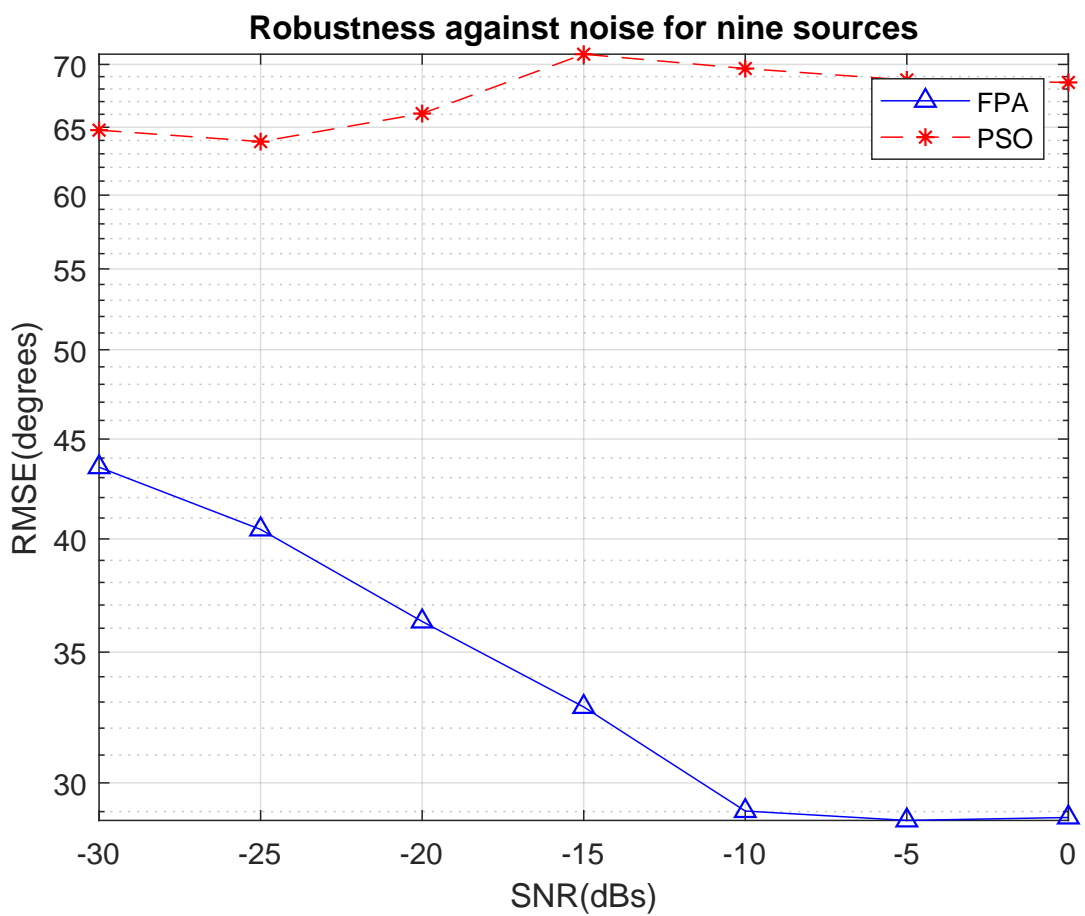

Figure 6. Robustness against noise for eleven targets.

\subsection{RMSE Analysis against Multiple Snapshots}

Based on Figures 4-6, the value of Root Mean Square Error (RMSE) is observed by increasing the Signal to Noise Ratio (SNR) from $-30 \mathrm{~dB}$ to $0 \mathrm{~dB}$ while in Figures 7-9 comparison of RMSE for FPA and PSO is performed with respect to a number of snapshots. In array signal processing, when direction of arrival estimation is performed multiple snapshots of the received signal are taken while we simulate the problem. If value of error reduced gradually when increasing the number of snapshots, it means that our algorithm works properly. Therefore, we perform the comparison of RMSE vs. snapshots for FPA and 
PSO in Figures 7-9 to explore the superiority of the algorithms . It can be clearly observed in Figures 7-9, that FPA is more effective for DOA estimation with enhanced degree of freedom than PSO. Moreover, as number of snapshots increases, RMSE decreases gradually.

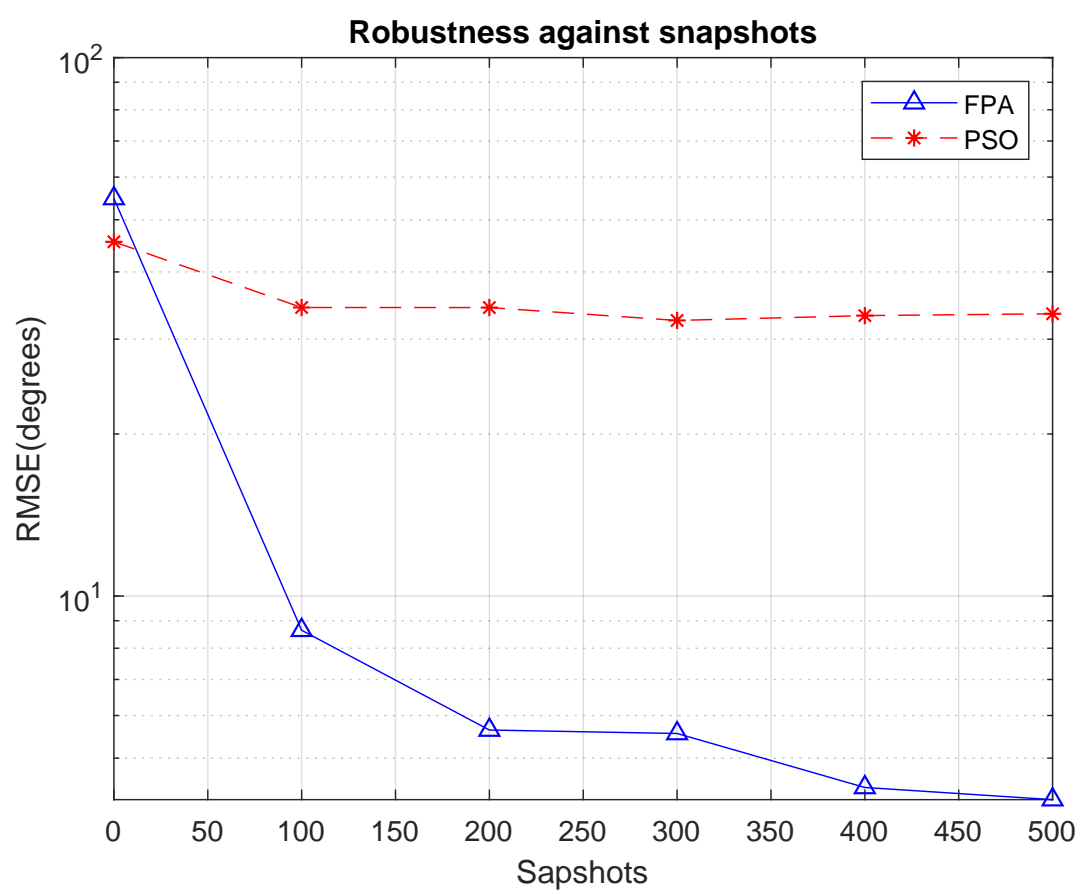

Figure 7. Robustness against snapshots for seven targets.

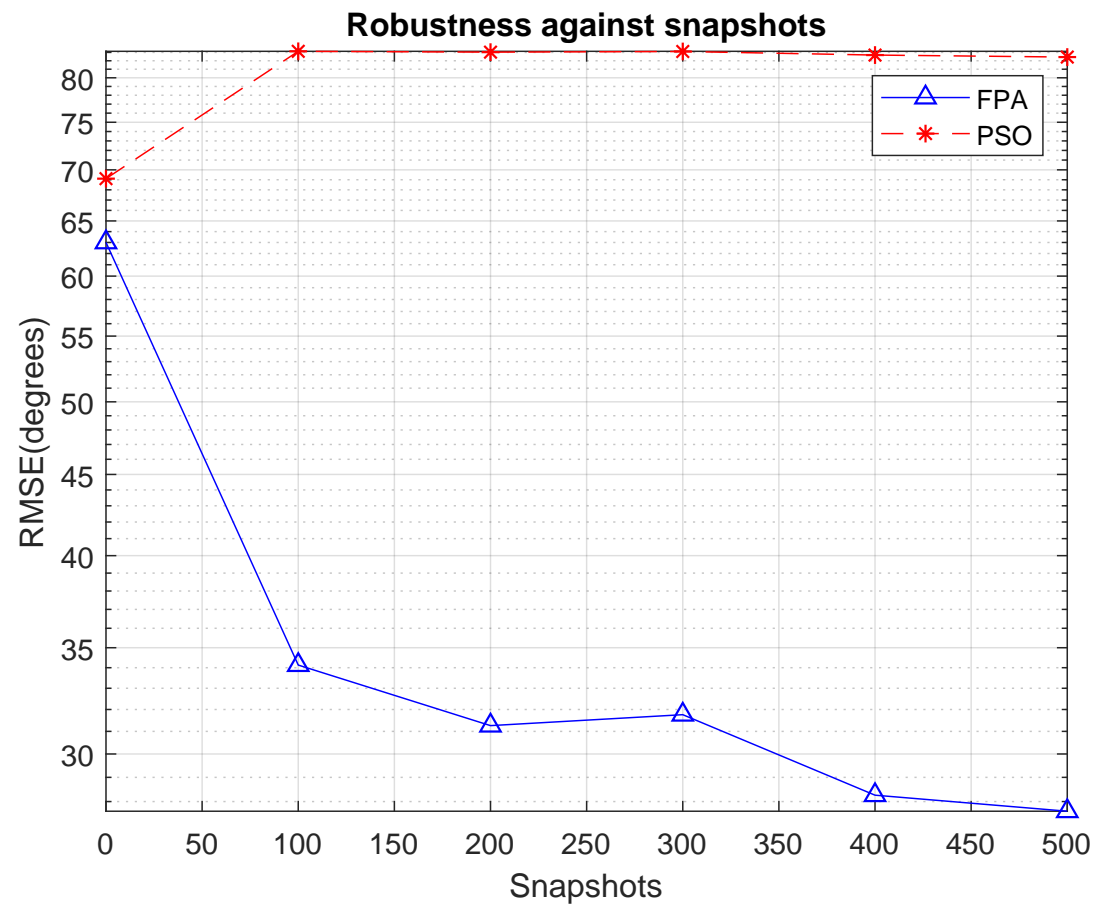

Figure 8. Robustness against snapshots for nine targets. 


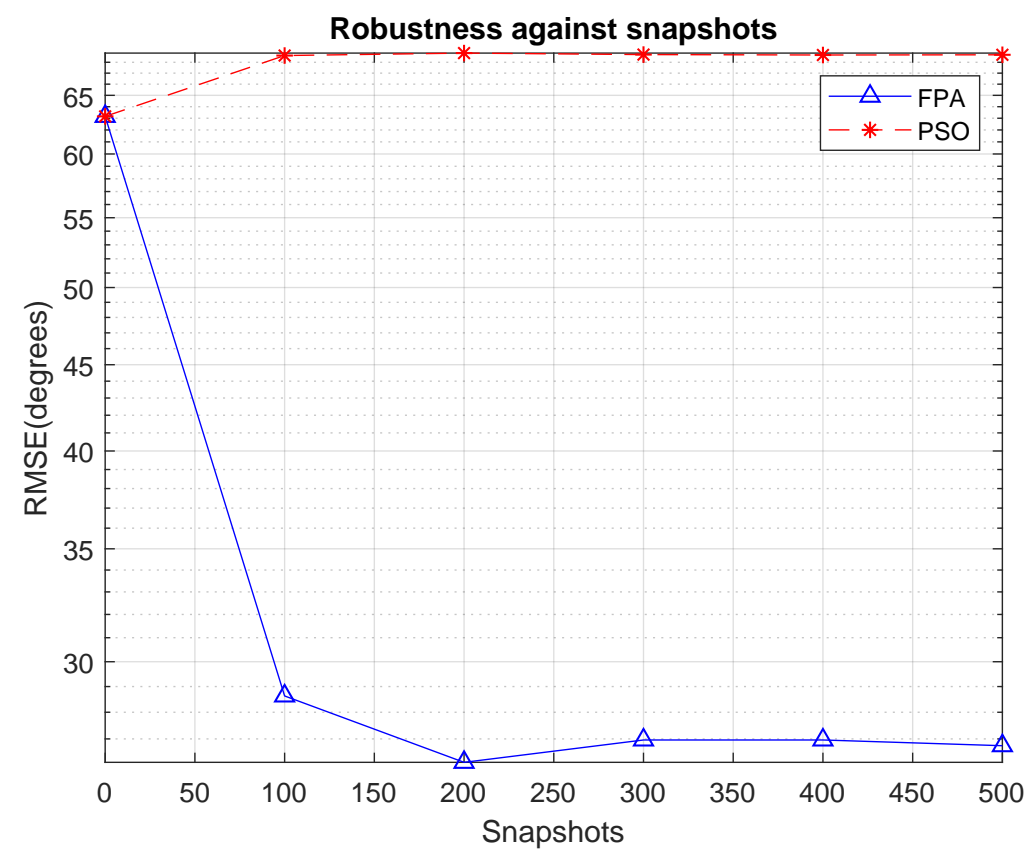

Figure 9. Robustness against snapshots for eleven targets.

\subsection{Variation Analysis of RMSE}

A box-plot is a standard way to demonstrate the distribution of error in accordance with $Q_{1}, Q_{2}, Q_{3}$, min and max value of data. We distinguish the min and max values on the basis of outlier and in conformity with outlier, we easily identify the performance of the proposed methodologies. In Figure 10 maximum RMSE of FPA is 40, 55 and 60 at $0 \mathrm{~dB},-5 \mathrm{~dB}$ and $-10 \mathrm{~dB}$, respectively. Whereas at $0 \mathrm{~dB},-5 \mathrm{~dB}$ and $-10 \mathrm{~dB}$ the relative maximum RMSE is below 400 and 600 . These plots showed that FPA performed vastly superior than PSO.
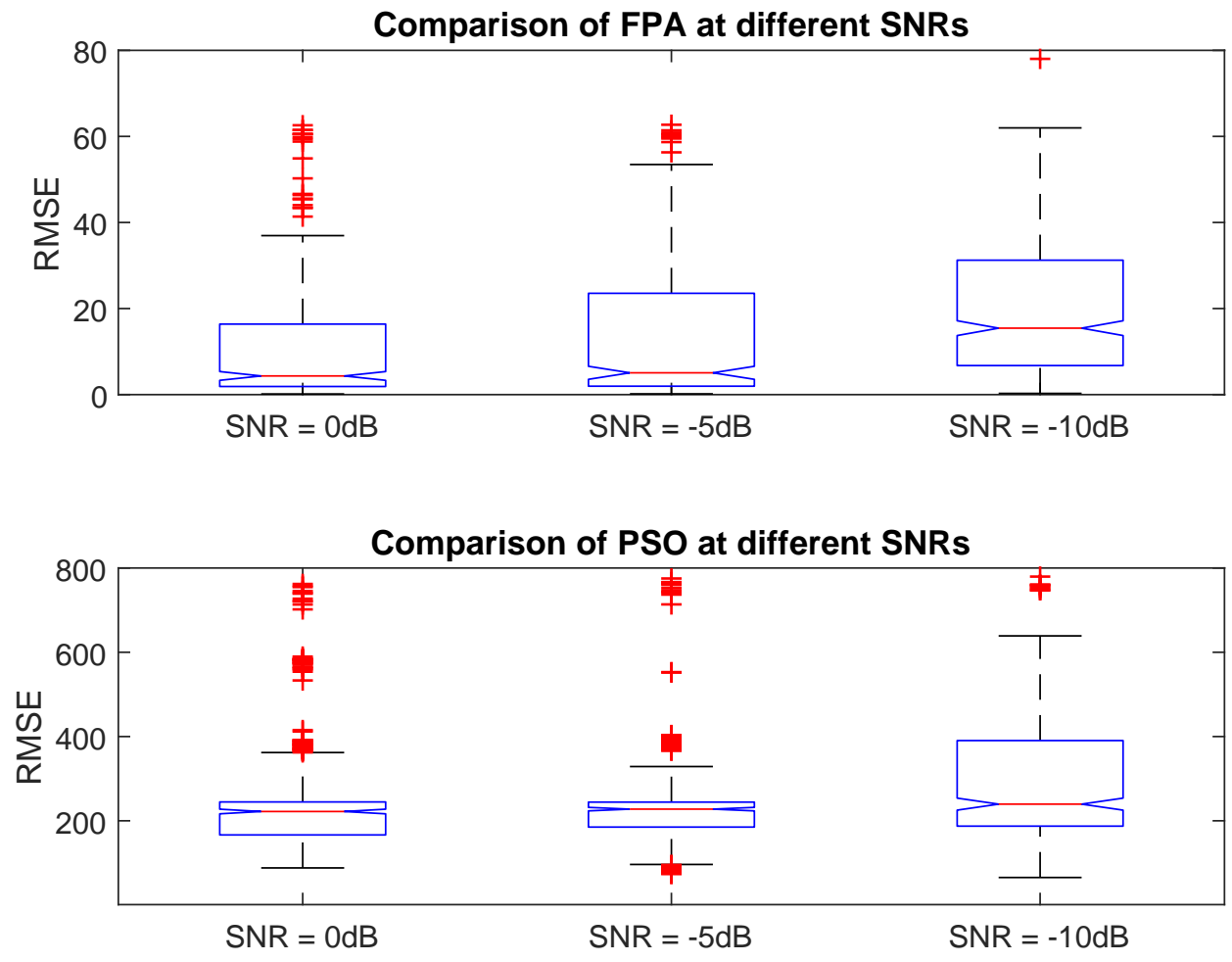

Figure 10. Variation analysis of RMSE for seven targets. 
In the case of nine targets, it can be easily observed that spread of RMSE of FPA is far less than PSO at $0 \mathrm{~dB},-5 \mathrm{~dB}$ and $-10 \mathrm{~dB}$ as depicted in Figure 11. In the case of eleven targets detection, again the performance of FPA is outclassed, as illustrated in Figure 12. In accordance with this diagram RMSE spread of FPA is below 200 at $0 \mathrm{~dB},-5 \mathrm{~dB}$ and $-10 \mathrm{~dB}$ while this spread is more than 1300 in case of PSO.
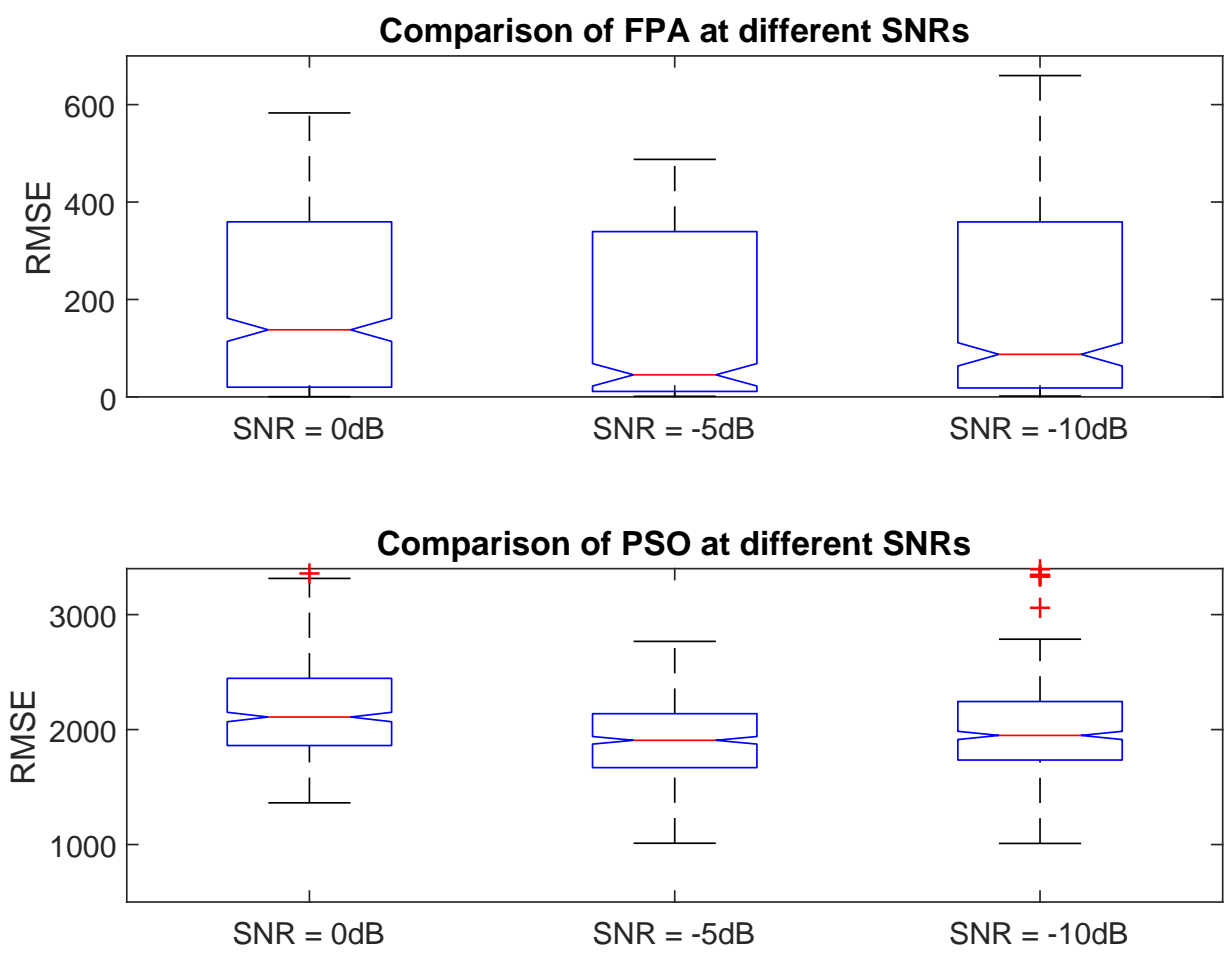

Figure 11. Variation analysis of RMSE for nine targets.
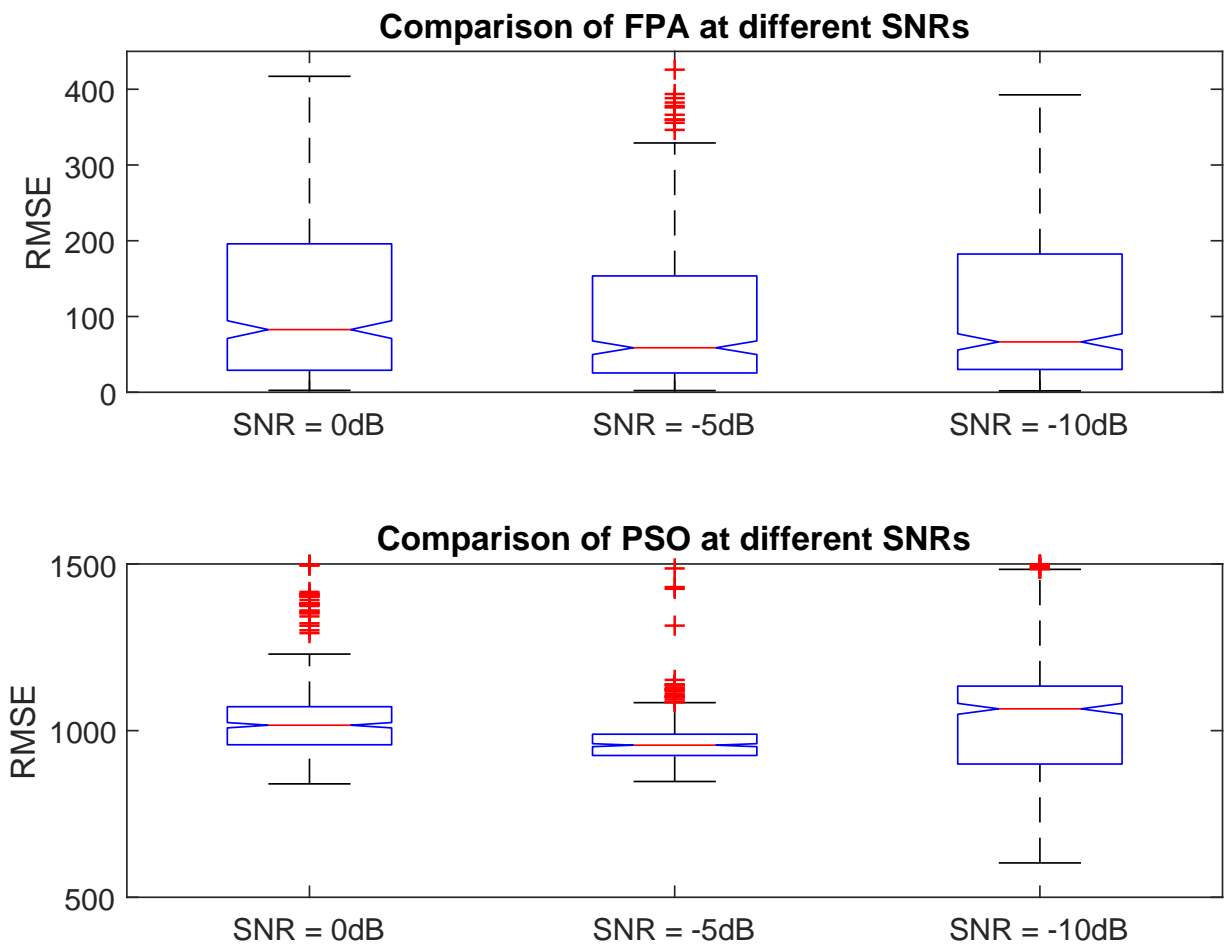

Figure 12. Variation analysis of RMSE for eleven targets. 


\subsection{Cumulative Distribution Function of RMSE}

$\mathrm{CDF}$ is a non decreasing function and in this subsection, it is applied to specify the persistence and failure times of optimization techniques in respect with Monte Carlo runs. $\mathrm{CDF}$ is the part of probability density function and it is presented in the form of probability. Figure 13 shows that FPA performs very well as compared to PSO in respect with RMSE. In case of FPA, RMSE has been occurred around zero at $80 \%$ of MC runs at $0 \mathrm{~dB},-5 \mathrm{~dB}$ and $-10 \mathrm{~dB}$ while RMSE is exceeded up to 150 at $25 \%$ of MC runs of PSO algorithm.
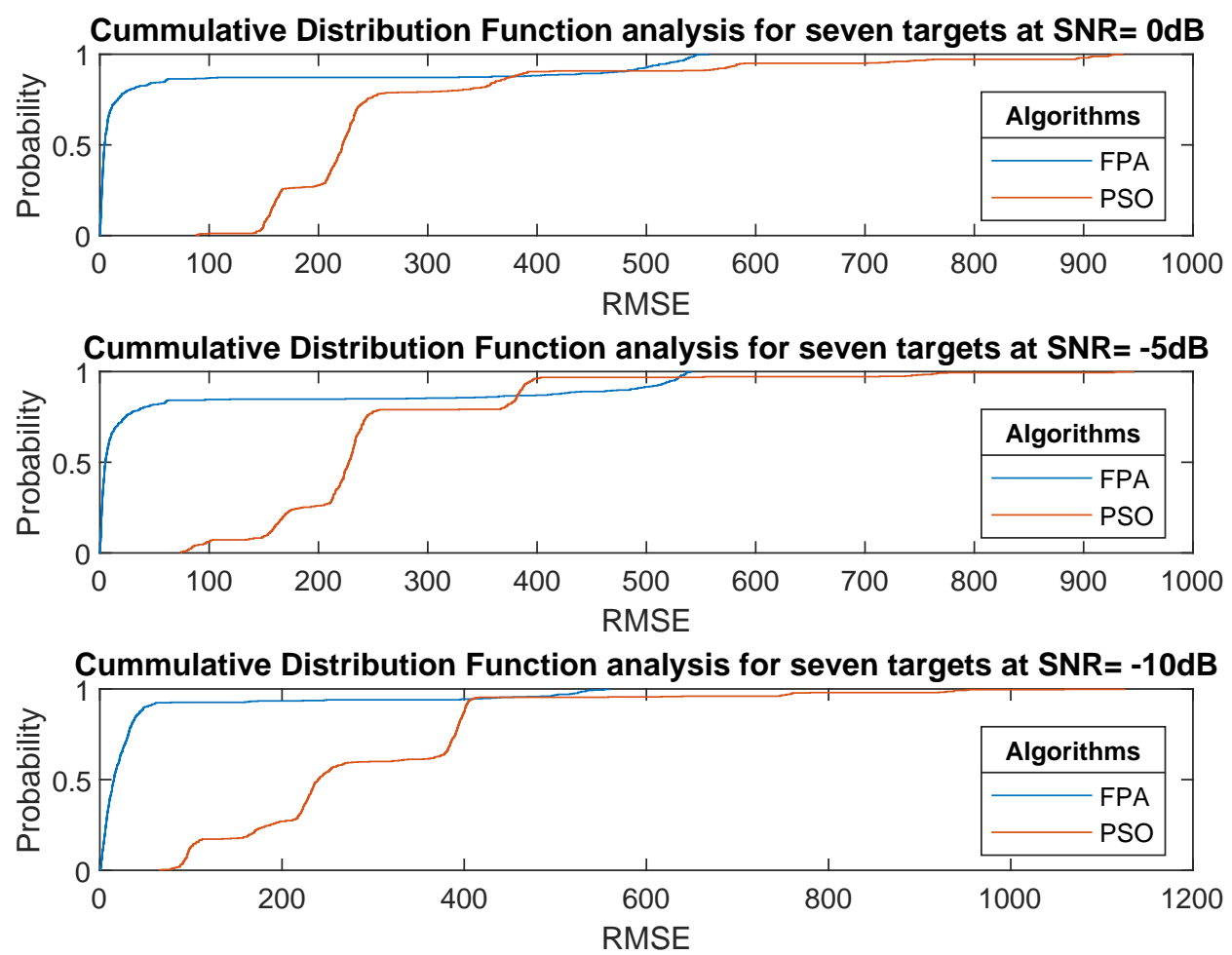

Figure 13. CDF analysis of RMSE for seven targets at different SNRs.

In case of nine targets estimation, again FPA performs superbly. The value of RMSE is near to zero at $50 \%$ of MC runs while it exceed up to 1500 in the scenario of PSO at $0 \mathrm{~dB}$, $-5 \mathrm{~dB}$ and $-10 \mathrm{~dB}$ as depicted in Figure 14.

In case of eleven targets estimation, the performance of FPA is remarkable. In this case, the RMSE value is near to 50 at $50 \%$ of MC runs in FPA algorithm while it exceed 1000 in the scenario of PSO at $0 \mathrm{~dB},-5 \mathrm{~dB}$ and $-10 \mathrm{~dB}$ as shown in Figure 15.

\subsection{Histogram Analysis of RMSE}

In histogram representation, bars are in the form of continuous grouping. In this analysis, we check how much RMSE occurred against Monte Carlo runs. On the basis of this analysis, we checked the performance of algorithms. In Figure 16 RMSE of FPA is very low at $0 \mathrm{~dB},-5 \mathrm{~dB}$ and $-10 \mathrm{~dB}$ SNR over 500 iterations. In the same scenario, RMSE of PSO increases gradually as SNR decreases. Thus, we clearly observed that FPA performed very well as compared to PSO on the basis of RMSE distribution.

In Figure 17, RMSE spread of FPA is very low at $0 \mathrm{~dB},-5 \mathrm{~dB}$ and $-10 \mathrm{~dB}$ SNR in accordance with 500 iterations while spread of RMSE of PSO is very wide. Thus, it can be noticed clearly that performance of is much better as compared to PSO on the basis of RMSE distribution.

As per Figure 18, RMSE spread of FPA is very small at $0 \mathrm{~dB},-5 \mathrm{~dB}$ and $-10 \mathrm{~dB}$ SNR with respect to 500 iterations while RMSE spread of PSO more wider. Thus, it can be examine easily that performance of FPA is much better as compared to PSO on the basis of RMSE distribution. 

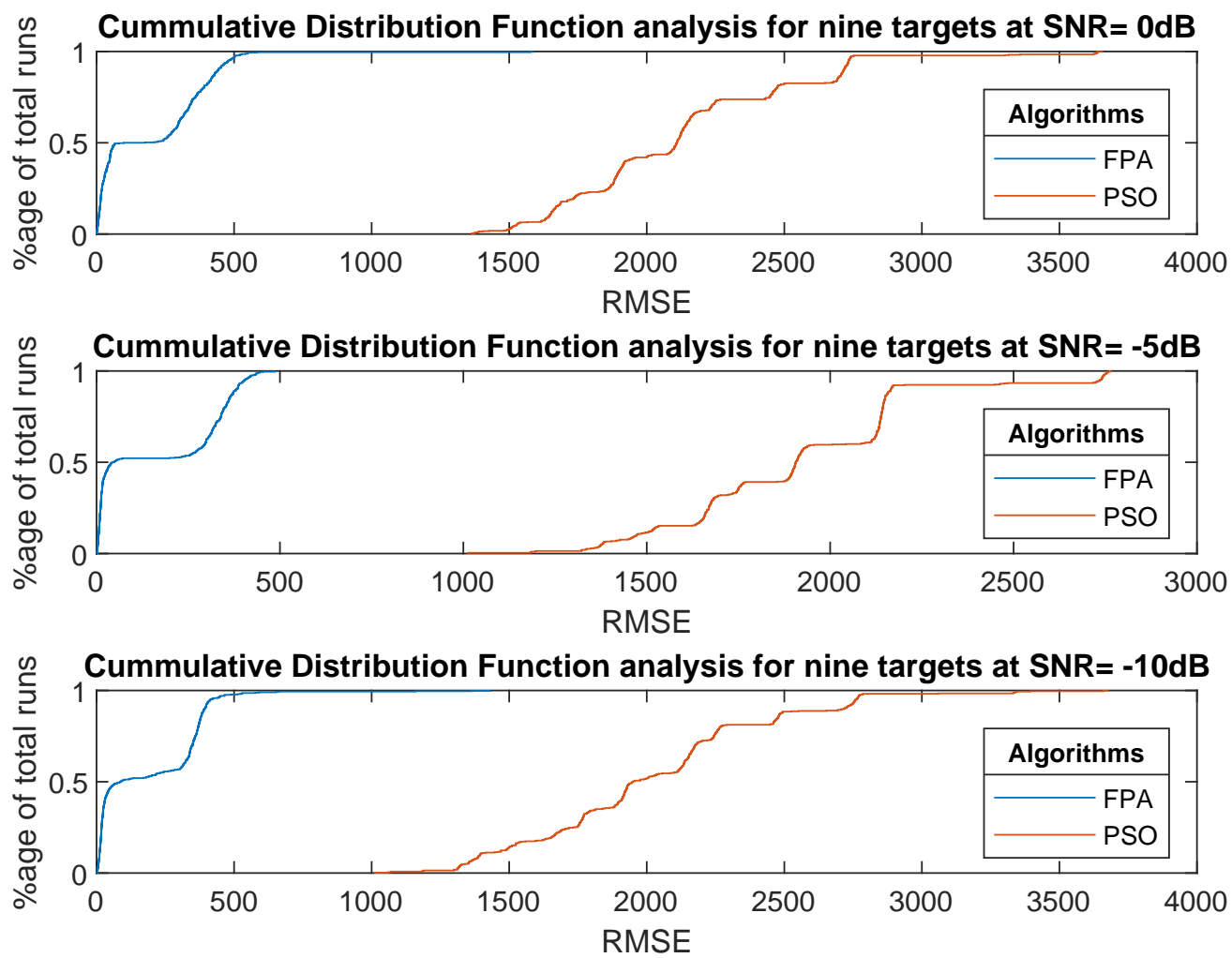

Figure 14. CDF analysis of RMSE for nine targets at different SNRs.
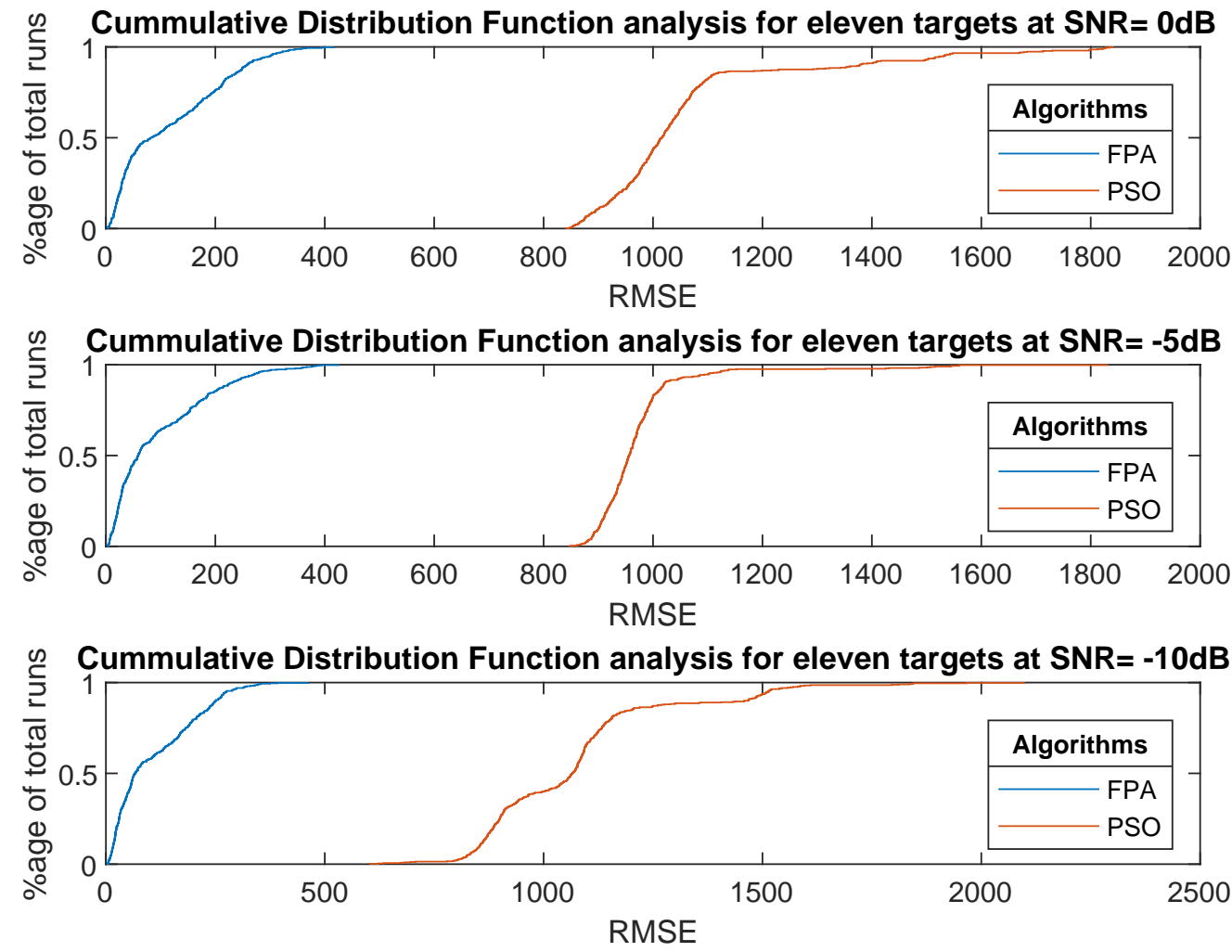

Figure 15. CDF analysis of RMSE for eleven targets at different SNRs. 

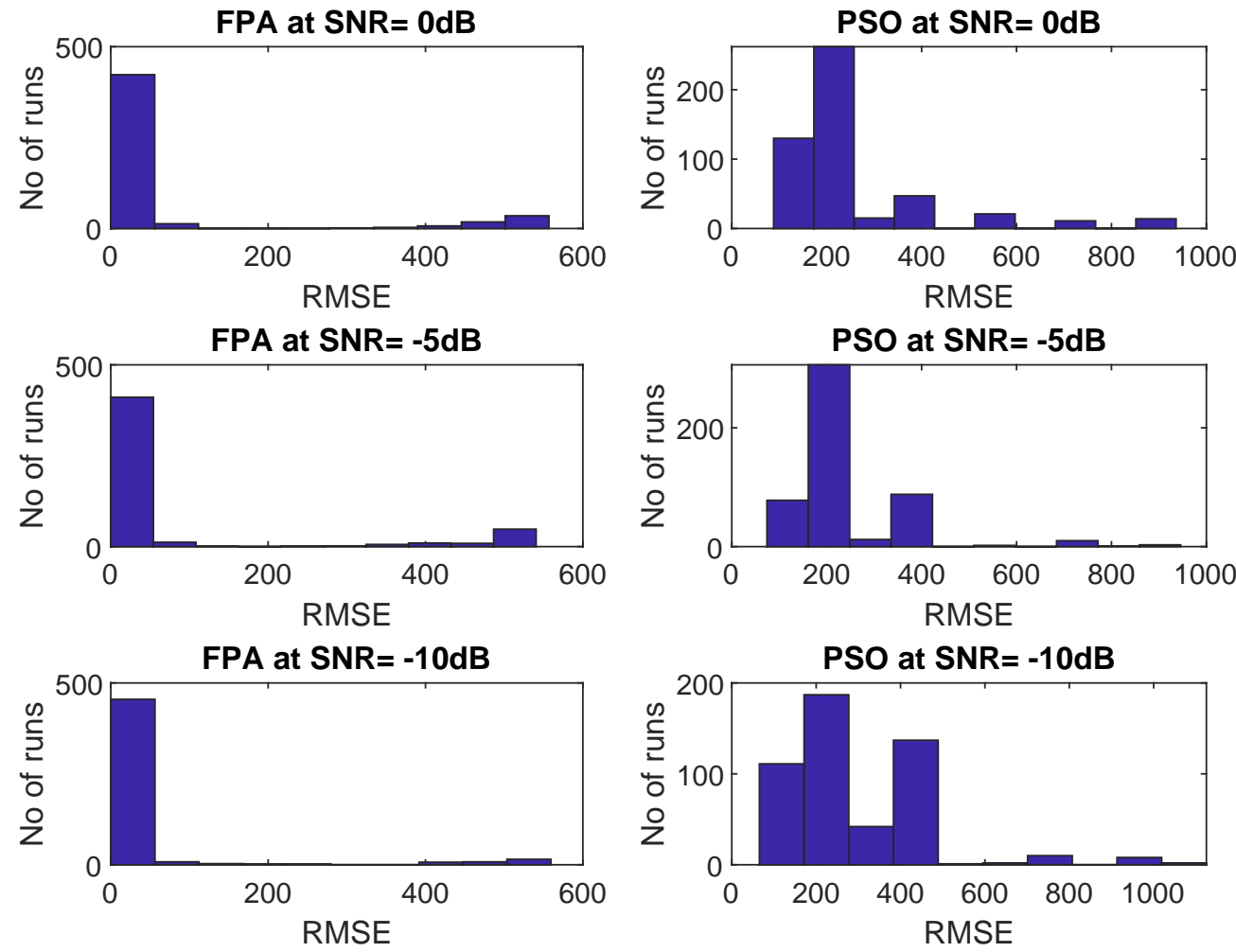

Figure 16. Histogram analysis of RMSE for seven targets.
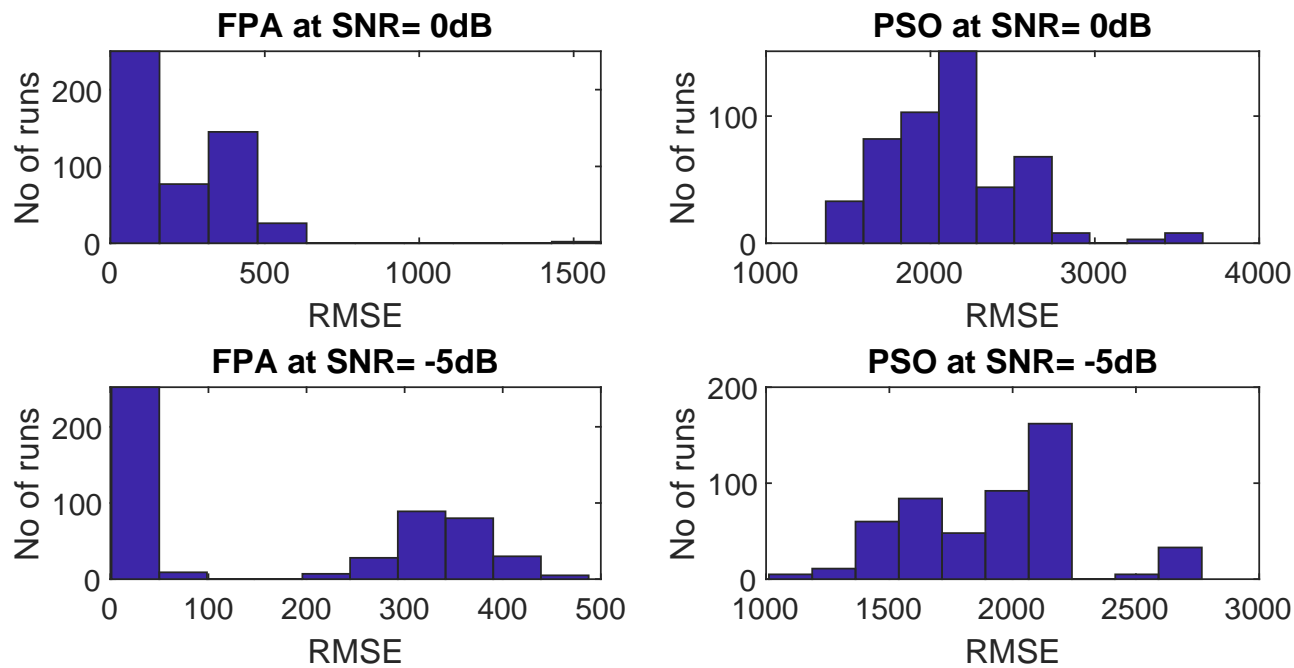

FPA at SNR $=-10 \mathrm{~dB}$
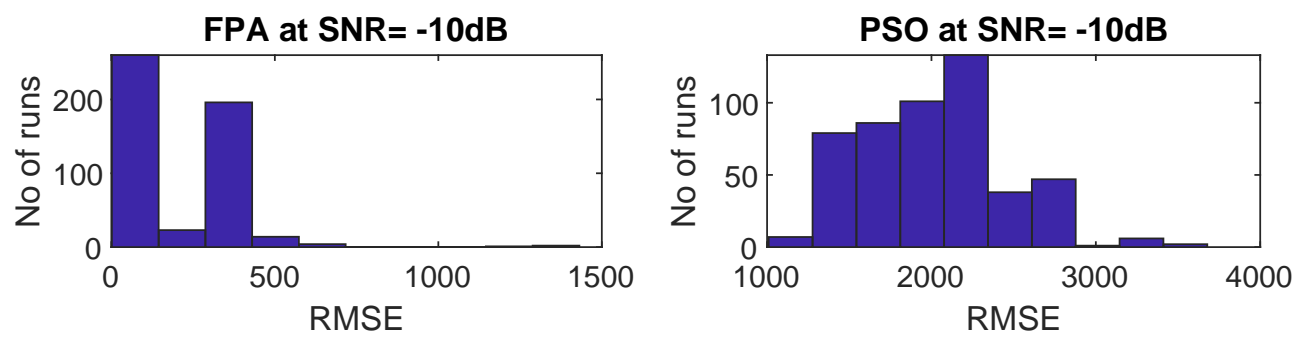

Figure 17. Histogram analysis of RMSE for nine targets. 

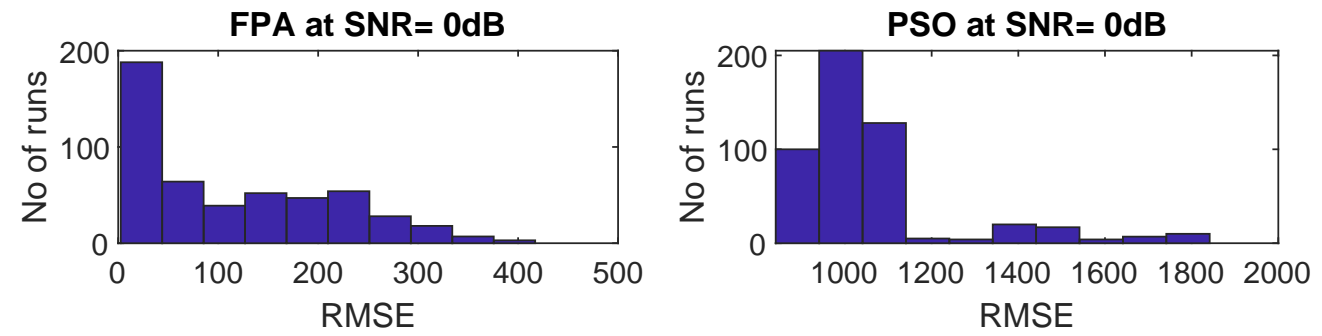

FPA at SNR $=-5 d B$
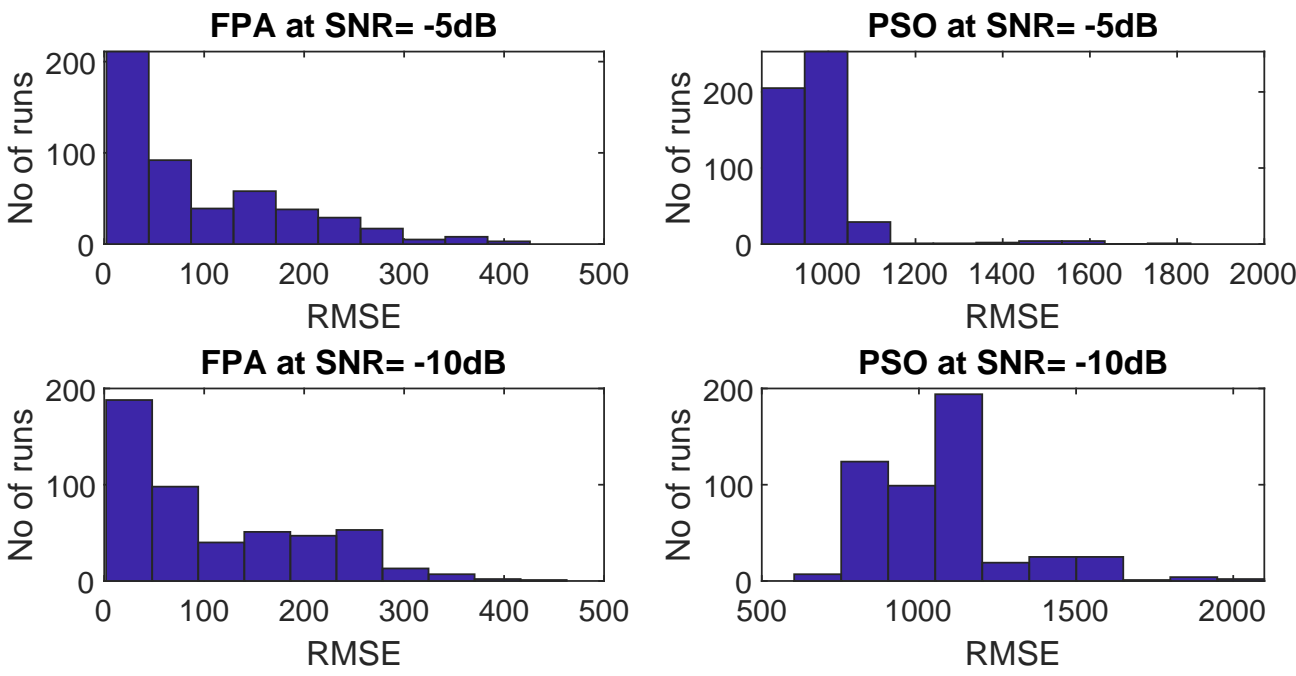

Figure 18. Histogram analysis of RMSE for eleven targets.

\section{Conclusions}

In this paper, we estimate DOA of electromagnetic waves received at coprime arrays through the Flower Pollination Algorithm. In DOA estimation, enhanced degree of freedom and estimation accuracy are the main challenges in a low SNR environment. Deterministic algorithms provide high resolution, but performance of these algorithms is degraded when we need higher DOF in a low SNR regime. However, meta heuristic algorithm, as we implement FPA in this paper, provides higher DOF and resolution ability at low SNR. In the future, we will expand the span of this study by estimating other parameters like frequency and amplitude of the impinging waves by exploring advance meta heuristic techniques.

Author Contributions: Conceptualization, K.H., N.A. (Nauman Ahmed), W.K. and S.T.; Methodology, K.H., N.A. (Nauman Ahmed), W.K., A.B., A.A. and F.S.A.; Validation, N.A. (Norah Alnaim), K.H., N.A. (Nauman Ahmed), W.K., A.B., A.A., F.S.A., M.S.Q. and F.A.; Analysis, K.H., N.A. (Nauman Ahmed), W.K. and A.B.; Writing-Original Draft Preparation, K.H.; Writing-Review and Editing, K.H., N.A. (Nauman Ahmed), W.K., A.B., A.A., F.S.A., M.S.Q. and F.A., Funding Acquisition, S.T., N.A. (Norah Alnaim), A.A. and F.S.A. All authors have read and agreed to the published version of the manuscript.

Funding: This work is supported in part by the Beijing Natural Science Foundation (No. 4212015), Natural Science Foundation of China (No. 61801008), China Ministry of Education - China Mobile Scientific Research Foundation (No. MCM20200102), China Postdoctoral Science Foundation (No. 2020M670074), Beijing Municipal Commission of Education Foundation (No. KM201910005025), China National Key Research and Development Program (No. 2018YFB0803600).

Institutional Review Board Statement: Not applicable.

Informed Consent Statement: Not applicable.

Data Availability Statement: The authors confirm that the data supporting the findings of this study are available within the article. 
Acknowledgments: We are immensely grateful to Florian Römer, Group Manager, Fraunhofer Institute for Nondestructive Testing IZFP (Ilmenau University of Technology Germany) for his unconditional help and guidance that greatly improved the manuscript. His expert opinion and assistance enable us to achieve this milestone. We would also like to state our gratitude to the late Ijaz Mansoor Qureshi for sharing his pearls of wisdom and care during the course of this research work. Although, he is no longer present among us, his guidance and love will always be there to show us the right path.

Conflicts of Interest: The authors declare no conflict of interest.

\section{References}

1. Zhang, Y.; Hu, G.; Zhu, M.; Zhan, C.; Zhao, F.; Zhou, H.; Zhao, F.; Yue, S. DOA estimation based on average processing of redundant virtual array elements for coprime MIMO RADAR. J. Phys. Conf. Ser. 2021, 1894, 012092. [CrossRef]

2. Wen, F.; Mao, C.; Zhang, G.. Direction finding in MIMO RADAR with large antenna arrays and non-orthogonal waveforms. Digit. Signal Process. 2019, 94, 75-83. [CrossRef]

3. Jing, H.; Wang, H.; Liu, Z.; Shen, X. DOA estimation for underwater target by active detection on virtual time reversal using a uniform linear array. Sensors 2018, 18, 2458. [CrossRef]

4. Straser, V.; Cataldi, D.; Cataldi, G. Radio direction finding system, a new perspective for global crust diagnosis. New Concepts Glob. Tectonics J. 2018, 6, 203-211.

5. Wang, J.J.-M.; Liu, J.; Pare, T.E., Jr.; Wu, T.; Bajko, G.; Hsu, Y.-P. Direction Finding and Ftm Positioning in Wireless Local Area Networks. U.S. Patent 10,484,814, 19 November 2019.

6. Suryavanshi, N.B.; Reddy, K.V.; Chandrika, V.R. Direction finding capability in bluetooth 5.1 standard. In International Conference on Ubiquitous Communications and Network Computing; Springer: Berlin/Heidelberg, Germany, 2019; pp. 53-65.

7. Qin, S.; Zhang, Y.D.; Amin, M.G.; Himed, B. DOA estimation exploiting a uniform linear array with multiple co-prime frequencies. Signal Process. 2017, 130, 37-46. [CrossRef]

8. Guo, M.; Zhang, Y.D.; Chen, T. DOA estimation using compressed sparse array. IEEE Trans. Signal Process. 2018, 66, 4133-4146. [CrossRef]

9. Elbir, A.M. Two-dimensional DOA estimation via shifted sparse arrays with higher degrees of freedom. Circuits Syst. Signal Process. 2019, 38, 5549-5575. [CrossRef]

10. Liu, K.; Zhang, Y.D. Coprime array-based DOA estimation in unknown nonuniform noise environment. Digit. Signal Process. 2018, 79, 66-74. [CrossRef]

11. Chen, C.-Y.; Vaidyanathan, P.P. Minimum redundancy mimo radars. In Proceedings of the 2008 IEEE International Symposium on Circuits and Systems, Seattle, WA, USA, 18-21 May 2008; pp. 45-48.

12. Pal, P.; Vaidyanathan, P.P. Nested arrays: A novel approach to array processing with enhanced degrees of freedom. IEEE Trans. Signal Process. 2010, 58, 4167-4181. [CrossRef]

13. Liu, J.; Zhang, Y.; Lu, Y.; Ren, S.; Cao, S. Augmented nested arrays with enhanced DOF and reduced mutual coupling. IEEE Trans. Signal Process. 2017, 65, 5549-5563. [CrossRef]

14. Vaidyanathan, P.P.; Pal, P. Sparse sensing with coprime arrays. In Proceedings of the 2010 Conference Record of the forty Fourth Asilomar Conference on Signals, Systems and Computers, Pacific Grove, CA, USA, 7-10 November 2010; pp. 1405-1409.

15. Zheng, Z.; Huang, Y.; Wang, W.; So, H.C. Direction-of-arrival estimation of coherent signals via coprime array interpolation. IEEE Signal Process. Lett. 2020, 27, 585-589. [CrossRef]

16. Adhikari, K.; Drozdenko, B. Symmetry-imposed rectangular coprime and nested arrays for direction of arrival estimation with multiple signal classification. IEEE Access 2019, 7, 153217-153229. [CrossRef]

17. Muhammad, M.; Li, M.; Abbasi, Q.H.; Goh, C.; Imran, M. Direction of arrival estimation using hybrid spatial cross-cumulants and root-music. In Proceedings of the 2020 14th European Conference on Antennas and Propagation (EuCAP), Copenhagen, Denmark, 15-20 March 2020; pp. 1-5.

18. Ning, Y.; Ma, S.; Meng, F.; Wu, Q. DOA estimation based on ESPRIT algorithm method for frequency scanning LWA. IEEE Commun. Lett. 2020, 24, 1441-1445. [CrossRef]

19. Hakam, A.; Shubair, R.M.; Salahat, E. Enhanced DOA estimation algorithms using MVDR and MUSIC. In Proceedings of the 2013 International Conference on Current Trends in Information Technology (CTIT), Dubai, United Arab Emirates, 11-12 December 2013; pp. 172-176.

20. Wang, P.; Kong, Y.; He, X.; Zhang, M.; Tan, X. An improved squirrel search algorithm for maximum likelihood DOA estimation and application for MEMS vector hydrophone array. IEEE Access 2019, 7, 118343-118358. [CrossRef]

21. Jaafer, Z.; Goli, S.; Elameer, A.S. Best performance analysis of DOA estimation algorithms. In Proceedings of the 20181 st Annual International Conference on Information and Sciences (AiCIS), Fallujah, Iraq, 20-21 November 2018; pp. $235-239$.

22. Vikas, B.; Vakula, D. Performance comparision of MUSIC and ESPRIT algorithms in presence of coherent signals for DOA estimation. In Proceedings of the 2017 International conference of Electronics, Communication and Aerospace Technology (ICECA), Coimbatore, India, 20-22 April 2017; Volume 2, pp. 403-405. 
23. Ahmed, N.; Wang, H.; Raja, M.A.Z.; Ali, W.; Zaman, F.; Khan, W.U.; He, Y. Performance analysis of efficient computing techniques for direction of arrival estimation of underwater multi targets. IEEE Access 2021, 9, 33284-33298. [CrossRef]

24. Yang, X.-S. Flower pollination algorithm for global optimization. In International Conference on Unconventional Computing and Natural Computation; Springer: Berlin/Heidelberg, Germany, 2012; pp. 240-249.

25. Qamar, M.S.; Tu, S.; Ali, F.; Armghan, A.; Munir, M.F.; Alenezi, F.; Muhammad, F.; Ali, A.; Alnaim, N. Improvement of Traveling Salesman Problem Solution Using Hybrid Algorithm Based on Best-Worst Ant System and Particle Swarm Optimization. Appl. Sci. 2021, 11, 4780. [CrossRef]

26. Hammed, K.; Ghauri, S.A.; Qamar, M.S. Biological inspired stochastic optimization technique (pso) for DOA and amplitude estimation of antenna arrays signal processing in RADAR communication system. J. Sens. 2016, 2016. [CrossRef]

27. Zhao, H.; Hou, Y.; Mao, X. A synthetic layout method for distributed Nested Circular Array based on Ant colony algorithm. In Proceedings of the IET International RADAR Conference (IET IRC 2020), Chongqing, China, 4-6 November 2020; pp. 955-959.

28. Parsa, S.A.H.; Zadeh, A.E.; Kazemitabar, S.J. A novel modified artificial bee colony for doa estimation. Int. J. Sens. Wirel. Commun. Control 2021, 11, 96-106. [CrossRef]

29. Jia, W.; Liu, S. Application of Simulated Annealing Genetic Algorithm in DOA estimation technique. Comput. Eng. Appl. 2014, 12, 266-270.

30. Das, S.; Suganthan, P.N. Differential Evolution: A survey of the state-of-the-art. IEEE Trans. Evol. Comput. 2010, 15, 4-31. [CrossRef]

31. Chen, H.; Li, H.; Yang, M.; Xiang, C.; Suzuki, M. General Improvements of Heuristic Algorithms for Low Complexity DOA Estimation. Int. J. Antennas Propag. 2019, 2019, 3858794. [CrossRef]

32. Sallam, T.A.R.; Abdel-Rahman, A.B.; Alghoniemy, M.; Kawasaki, Z. Flower Pollination Algorithm for Adaptive Beamforming of Phased Array Antennas. J. Mach. Intell. 2017, 2 , 1-5. [CrossRef]

33. Pal, P.; Vaidyanathan, P.P. Coprime sampling and the MUSIC algorithm. In Proceedings of the 2011 Digital Signal Processing and Signal Processing Education Meeting (DSP/SPE), Sedona, AZ, USA, 4-7 January 2011; IEEE: Piscataway, NJ, USA, 2011; pp. 289-294. 